\title{
H I observations of galaxies
}

\section{The Coma Supercluster ${ }^{\star}$}

\author{
G. Gavazzi ${ }^{1}$, K. O’Neil ${ }^{2}$, A. Boselli ${ }^{3}$, and W. van Driel ${ }^{4}$
}

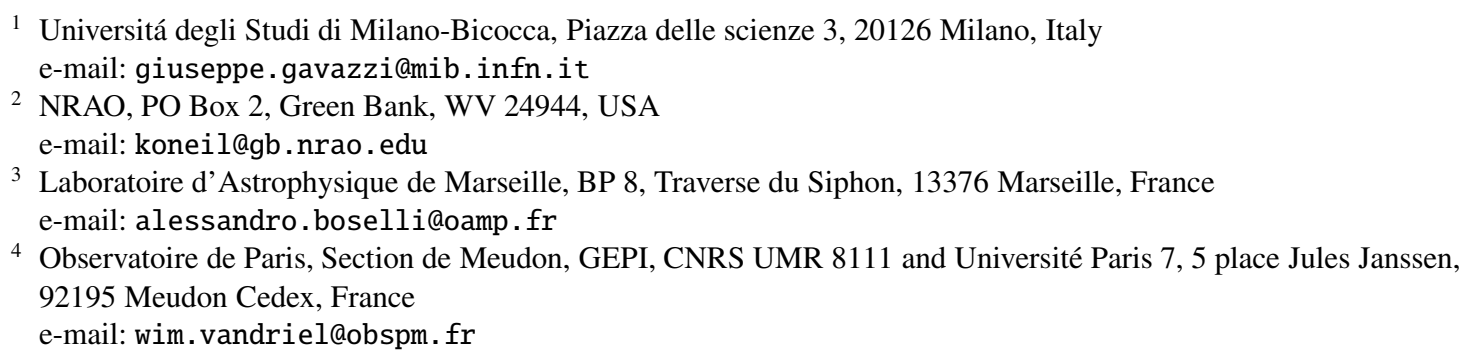

Received 18 July 2005 / Accepted 20 September 2005

\section{ABSTRACT}

High sensitivity $21-\mathrm{cm} \mathrm{H}$ I line observations with an rms noise level of $\sim 0.5 \mathrm{mJy}$ were made of 35 spiral galaxies in the Coma Supercluster, using the refurbished Arecibo telescope, leading to detection of 25 objects. These data, combined with the measurements available in the literature, provide the set of $\mathrm{HI}$ data for $94 \%$ of all late-type galaxies in the Coma Supercluster with an apparent photographic magnitude $m_{\mathrm{p}} \leq 15.7 \mathrm{mag}$. We confirm that the typical scale of $\mathrm{H}$ I deficiency around the Coma cluster is $2 \mathrm{Mpc}$, i.e. one virial radius. Comparing the H I mass function (HIMF) of cluster with non-cluster members of the Coma Supercluster we detected a shortage of high H I mass galaxies among cluster members that can be attributed to the pattern of H I deficiency found in rich clusters.

Key words. galaxies: distances and redshifts - galaxies: general - galaxies: ISM - galaxies: clusters: individual: Virgo radio lines: galaxies

\section{Introduction}

H I line observations of galaxies have provided us with some of the most powerful diagnostics on the role of the environment in regulating the evolution of late-type (spiral) galaxies in the local Universe. Spiral galaxies in rich X-ray luminous clusters display a significant lack of $\mathrm{H}$ I gas with respect to their "undisturbed" counterparts in the field (Haynes \& Giovanelli 1984; Giovanelli \& Haynes 1985). This pattern of H I deficiency can be attributed to various interaction mechanisms: ram-pressure (Gunn \& Gott 1972), viscous stripping (Nulsen 1982), thermal evaporation (Cowie \& Songaila 1977), or tidal interaction with the cluster potential well (Byrd \& Valtonen 1990; Moore et al. 1996). Since these mechanisms have a higher efficiency in or near rich cluster cores, the H I deficiency parameter (Haynes \& Giovanelli 1984 - see Sect. 5.1) is an environmental indicator that provides a clear signature of a galaxy's membership in a rich cluster.

* Table 4, Appendix A, Figs. A.1 and A.2 are only available in electronic form at http://www. edpsciences.org
The Coma Supercluster has received considerable attention in H I studies due to its proximity to us $(\sim 100 \mathrm{Mpc})$. Since the pioneering study by Sullivan et al. (1981), various works (e.g. Chincarini et al. 1983a; Gavazzi 1987, 1989; Scodeggio \& Gavazzi 1993; Haynes et al. 1997) have provided measurements of the H I content for most late-type galaxies in the Coma Supercluster. In addition to these single-dish studies of their global H I properties, the detailed mapping of galaxies in the Coma and A1367 clusters with radio synthesis telescopes was obtained by Bravo-Alfaro et al. $(2000,2001)$ and Dickey \& Gavazzi (1991).

A high sensitivity, blind H I survey of 7000 square deg. of sky is planned for 2005-2006 with the ALFA multibeam system at Arecibo (Giovanelli et al. 2005), and even more sensitive (1 mJy rms) surveys of parts of these clusters will be obtained with the ALFA system. They will include the Coma Supercluster and the Virgo cluster. In preparation for these surveys, before the installation of the ALFA system, we used the single-beam Arecibo system to continue the pointed observation survey of late-type galaxies in the Virgo cluster (see Gavazzi et al. 2005a, Paper I) and in the Coma Supercluster 


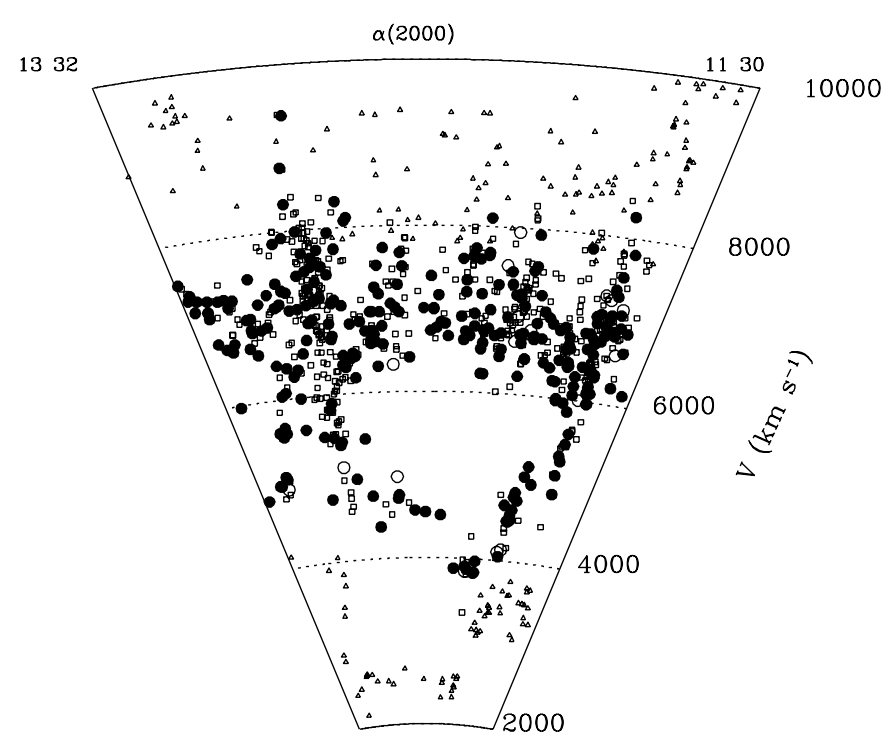

Fig. 1. The wedge diagram of the Coma Supercluster galaxies in the declination interval $18^{\circ}<\delta<32^{\circ}$. Members are divided between late-type (large circles, filled if observed in HI) and early-type (E-SOa: empty squares). Empty triangles mark foreground and background galaxies.

area. Here we report the results of the Coma Supercluster observations that, in conjunction with the previously available H I data-set, enable us to review the properties of galaxies in this Supercluster, as obtained from optically selected H I observations.

The selection of the cluster targets for $\mathrm{HI}$ observations is described in Sect. 2, the observations and the data reduction are presented in Sect. 3 and the results are given in Sect. 4 and discussed in Sect. 5. A Hubble constant of $75 \mathrm{~km} \mathrm{~s}^{-1} \mathrm{Mpc}^{-1}$ is assumed throughout this paper.

\section{Sample selection}

Galaxies in the present study were selected from the CGCG Catalogue (Zwicky et al. 1961-68) in the region $11^{\mathrm{h}} 30^{\mathrm{m}}<\alpha<13^{\mathrm{h}} 30^{\mathrm{m}} ; 18^{\circ}<\delta<32^{\circ}$. There are 1127 CGCG galaxies listed in this region with an apparent photographic magnitude $m_{\mathrm{p}} \leq 15.7$. Their wedge-diagram is given in Fig. 1, where the structure of the Coma-A1367 Supercluster stands out clearly as the pronounced density enhancement near $7000 \mathrm{~km} \mathrm{~s}^{-1}$ and as part of one of the largest known coherent structures in the local universe, named the "Great Wall" (Ramella et al. 1992). Other conspicuous features are: (i) the "Fingers of God" of Coma and A1367, that span the interval $4000<V<10000 \mathrm{~km} \mathrm{~s}^{-1}$, mostly traced by earlytype objects, and (ii) the large $\left(\sim 7500 \mathrm{Mpc}^{3}\right)$ "void" in front of the Supercluster, with a density that is 150 times lower than the mean galaxy density in the universe. Other remarkable features, also known as the "legs of the homunculus" (de Lapparent et al. 1986) are two filaments pointing toward the Coma cluster in the interval $4500<V<7000 \mathrm{~km} \mathrm{~s}^{-1}$. A third "homunculus leg" surrounds the void on the western side, projected near A1367. Objects in the interval $6000<V<8000 \mathrm{~km} \mathrm{~s}^{-1}$ form a bridge between the two clusters with a narrow velocity distribution.

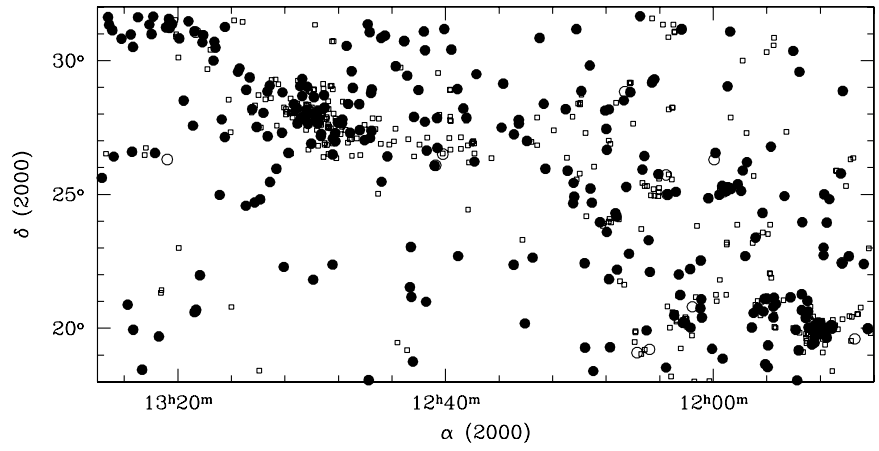

Fig. 2. The sky projection of Coma Supercluster members (including the "homunculus legs" HL). Spirals are represented by circles (filled if observed in $\mathrm{HI}$ ) and E-SOa objects by empty squares.

Above $V>8000 \mathrm{~km} \mathrm{~s}^{-1}$, the Coma Supercluster fades into the background, so setting a boundary between its members and the projected background objects is rather arbitrary. In assigning the membership of individual galaxies to the various substructures we follow the criteria of Gavazzi et al. (1999).

Out of the 1127 galaxies in Fig. 1, 654 are considered proper Supercluster members according to these criteria, and 76 additional galaxies belonging to the "homunculus legs" (HL) are considered separately (see Table 1). Their sky projection is given in Fig. 2, revealing a substantial morphology segregation between late-type (spiral) and early-type (E-SOa) objects. The Coma Supercluster has also been observed in $\mathrm{H} \mathrm{I}$ with remarkable completeness: $65 \%$ of the published data are found in 5 publications: Chincarini et al. (1983a), Gavazzi (1987, 1989), Scodeggio \& Gavazzi (1993), Haynes et al. (1997).

After the present high sensitivity ( $\mathrm{rms} \sim 0.5 \mathrm{mJy}$ ) observations of 33 additional CGCG galaxies (and of two fainter objects: FOCA 610, 636), 295/315 (94\%) late-type members (including HL) were observed (see Table 1), of which 259 were detected and 36 have upper limits. Of the remaining 20 unobserved late-type targets, 13 are members of double or multiple systems that could not be resolved by the Arecibo beam (97-111S, 97-129E, 127-051N, 128-029E, $127-025 \mathrm{~N}, 159-049 \mathrm{~S}, 128-031 \mathrm{~N}, 128-002$, 97-036, 98-072, 98-073, 98-081, 98-087). The Coma cluster (160-243) and 6 galaxies (127-121, 129-016, 157-077, 158-046, 160-180, 161-029) belonging to the HL were not observed due to scheduling constraints. To fill in a scheduling hole, 13 galaxies in the Virgo cluster were also observed, and all but one were detected. The results of these observations are given in Table 3, and the HI profiles of the detected galaxies are shown in Fig. A.2. These objects will not be considered further in this paper.

All data on the Coma Supercluster and Virgo galaxies are collected and made available worldwide via the "Goldmine" website (http: //Goldmine . mib . infn . it; see Gavazzi et al. 2003).

\section{Observations}

Using the refurbished 305-m Arecibo Gregorian radio telescope, we observed 35 galaxies in the Coma Supercluster 
Table 1. Sample completeness.

\begin{tabular}{lllll}
\hline \hline & \multicolumn{2}{c}{ Type $<$ Sa } & \multicolumn{2}{c}{ Type $\geq$ Sa } \\
& All & H I & All & H I \\
\hline Coma members & 391 & 58 & 263 & 249 \\
HL & 24 & 10 & 52 & 46 \\
Background & 131 & 5 & 118 & 55 \\
Foreground & 46 & 16 & 102 & 92 \\
All & 592 & 89 & 535 & 442 \\
\hline
\end{tabular}

(plus 13 in the Virgo cluster) (see Sect. 2) in February 2004 and January-March 2005. Data were taken with the $L$-band Wide receiver, using a nine-level sampling with two of the 2048 lag subcorrelators set to each polarization channel. All observations were taken using the position-switching technique, with each blank sky (or OFF) position observed for the same duration, and over the same portion of the telescope dish as the on-source $(\mathrm{ON})$ observation. Each $5 \mathrm{~min}+5 \mathrm{~min}$ $\mathrm{ON}+\mathrm{OFF}$ pair was followed by a $10 \mathrm{~s} \mathrm{ON+OFF}$ observation of a well-calibrated noise diode. The overlaps between both sub-correlators with the same polarization allowed a contiguous radial velocity search range of sufficient width from -1000 to $8500 \mathrm{~km} \mathrm{~s}^{-1}$. The velocity resolution was $2.6 \mathrm{~km} \mathrm{~s}^{-1}$, the instrument's $H P B W$ at $21 \mathrm{~cm}$ is $3.5 \times 3.1$ and the pointing accuracy was about $15^{\prime \prime}$. The pointing positions used were the optical center positions of the target galaxies listed in Table 1. Flux density calibration corrections were good to within $10 \%$ (and often much better); see the discussion of the errors involved in O’Neil (2004).

Using standard IDL data reduction software available at Arecibo, corrections were applied for the variations in the gain and system temperature with zenith angle and azimuth. A baseline of order one to three was fitted to the data, excluding those velocity ranges with $\mathrm{HI}$ line emission or radio frequency interference (RFI). The velocities were corrected to the heliocentric system, using the optical convention, and the polarizations were averaged. All data were boxcar-smoothed to a velocity resolution of $12.9 \mathrm{~km} \mathrm{~s}^{-1}$ for further analysis. For all spectra, the rms noise level was determined and for the detected objects the central line velocity, the line widths at, respectively, the $50 \%$ and $20 \%$ level of the peak, and the integrated line flux were determined. No flux correction depending on the source size was applied because the optical extent of all detected targets does not significantly exceed the Arecibo $H P B W$.

\section{Results}

In order to identify those sources whose H I detections could have been confused by nearby galaxies, we queried the NED, HyperLeda, and Goldmine databases and inspected DSS images over a region of $10^{\prime}$ radius surrounding the central position of each source, given the telescope's sidelobe pattern. Quoted values are weighted averages from the HyperLeda database, unless otherwise indicated.

The H I spectra of both the clearly and the marginally detected galaxies are shown in Figs. A.1 and A.2, and the global
H I line parameters are listed in Table 3 . These are directly measured values; no corrections have been applied to them for, e.g., instrumental resolution. Table 3 is organized as follows:

Col. 1: Obj. is the galaxy designation;

Cols. 2-3: (J2000) celestial coordinates;

Col. 4: the heliocentric optical recessional velocity (in $\mathrm{km} \mathrm{s}^{-1}$ );

Col. 5: the rms dispersion in the baseline (mJy/beam);

Col. 6: $S_{\mathrm{p}}$ is the peak flux density of the detected line (mJy/beam);

Col. 7: $\quad V_{\mathrm{HI}}$ is the heliocentric central radial velocity of a line profile (in $\mathrm{km} \mathrm{s}^{-1}$ ) in the optical convention with its estimated uncertainty (see below);

Cols. 8-9: $W_{50}$ and $W_{20}$ are the line widths at $50 \%$ and $20 \%$ of peak maximum, respectively, $\left(\mathrm{km} \mathrm{s}^{-1}\right)$;

Col. 10: $\quad I_{\mathrm{HI}}$ is the integrated line flux $\left(\mathrm{Jy} \mathrm{km} \mathrm{s}^{-1}\right)$ with its estimated uncertainty (see below);

Col. 11: A quality flag to the spectra is given, where $Q=1$ stands for high signal-to-noise, double-horned profiles; $Q=2$ for high signal-to-noise, single-horned profiles; and $Q=3,4$ for low signal-to-noise profiles whose measured line parameters are not reliable. $Q=5$ is given to unpublished profiles.

We estimated the uncertainties $\sigma_{V_{\mathrm{HI}}}\left(\mathrm{km} \mathrm{s}^{-1}\right)$ in $V_{\mathrm{HI}}$ and $\sigma_{I_{\mathrm{HI}}}\left(\mathrm{Jy} \mathrm{km} \mathrm{s}^{-1}\right)$ in $I_{\mathrm{HI}}$ following Schneider et al. (1986, 1990), as:

$\sigma_{V_{\mathrm{HI}}}=1.5\left(W_{20}-W_{50}\right) X^{-1}$

and

$\sigma_{I_{\mathrm{HI}}}=2\left(1.2 W_{20} / R\right)^{0.5} R \sigma=7.9\left(W_{20}\right)^{0.5} \sigma$

where $I_{\mathrm{HI}}$ is the integrated line flux $\left(\mathrm{Jy} \mathrm{km} \mathrm{s}^{-1}\right), R$ the instrumental resolution $\left(12.9 \mathrm{~km} \mathrm{~s}^{-1}\right)$, and $X$ the signal-to-noise ratio of a spectrum, i.e. the ratio of the peak flux density $S_{\mathrm{p}}$ and $\sigma$, the rms dispersion in the baseline (Jy). The uncertainties in the $W_{20}$ and $W_{50}$ line widths are expected to be 2 and 3 times $\sigma_{V_{\mathrm{HI}}}$, respectively.

\section{Discussion}

The newly obtained H I data were combined with those available from the literature for the $m_{\mathrm{p}} \leq 15.7$ late-type galaxies in the Coma Supercluster, as listed in Table 4. The sample comprises 315 galaxies, of which 295 were observed, 259 were detected, and 36 remain undetected. Table 4 is organized as follows:

Col. 1: galaxy designation in the CGCG Catalog;

Col. 2: morphological type;

Col. 3: apparent photographic magnitude from the CGCG;

Col. 4: membership as defined in Gavazzi et al. (1999). Distances $D$ of 96.0 and $91.3 \mathrm{Mpc}$ are assumed for Coma and A1367, respectively. Distances of individual groups and substructures in the Great Wall (including HL) are taken from Gavazzi et al. (1999) (rescaled to $H_{0}=75 \mathrm{~km} \mathrm{~s}^{-1} \mathrm{Mpc}^{-1}$ ). Distances from individual redshifts are assumed for supercluster isolated galaxies and members of multiplets; 


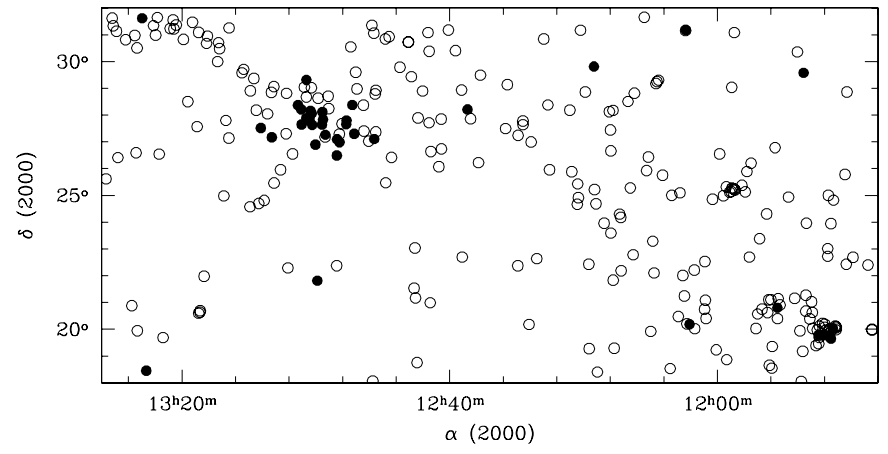

Fig. 3. The late-type Coma Supercluster members (including HL) coded according to their $\mathrm{HI}$ deficiency parameter: Def $_{\mathrm{HI}} \leq 0.5$ (empty circles); $\operatorname{Def}_{\mathrm{HI}}>0.5$ (filled circles).

Col. 5: recessional velocity in $\mathrm{km} \mathrm{s}^{-1}$;

Col. 6: HI mass or mass limit in solar units: $M_{\mathrm{HI}}=2.36 \times$ $10^{5} D^{2} I_{\mathrm{HI}}$. For undetected galaxies, we set $I_{\mathrm{HI}}=1.5 \times$ $\mathrm{rms}_{\mathrm{HI}} \times W_{\langle 20-50\rangle}$, where $\mathrm{rms}_{\mathrm{HI}}$ is the rms of the spectra in mJy, and the $W_{\langle 20-50\rangle}$ profile width is based on the following average line widths of the detected objects per Hubble type bin: $300 \mathrm{~km} \mathrm{~s}^{-1}$ for Sa-Sbc, $190 \mathrm{~km} \mathrm{~s}^{-1}$ for Sc-Scd;

Col. 7: coded reference to the H I measurement (see the notes to the table);

Col. 8: HI deficiency parameter as defined in Haynes \& Giovanelli (1984) (see Sect. 5.1);

Col. 9: quality flag (see last column of Table 3).

\subsection{The pattern of H I deficiency}

For the late-type galaxies in the present study, we estimated the HI deficiency parameter following Haynes \& Giovanelli (1984) as the logarithmic difference between $M_{\mathrm{HI}}$ of a reference sample of isolated galaxies and $M_{\mathrm{HI}}$ as actually observed in individual objects: $\operatorname{Def}{ }_{\mathrm{HI}}=\log M_{\mathrm{HI}}$ ref. $-\log M_{\mathrm{HI} \text { obs. }}$. Then, $M_{\mathrm{HI}}$ ref is computed from the galaxies optical linear diameter $d$ as: $\log M_{\mathrm{HI}}$ ref $=a+b \log (d)$, where $d$ is estimated consistently with Haynes \& Giovanelli (1984); and $a$ and $b$ are weak functions of the Hubble type, as listed in Table 3 of Paper I (notice that $b \sim 2$ across the Hubble sequence, i.e. $M_{\mathrm{HI}}$ ref increases approximately as the galaxy linear diameter squared).

Figure 3 shows that $\mathrm{H}$ I deficient galaxies segregate around the center of the Coma cluster and, to a much lesser degree, around A1367. One important issue that can be addressed with the present data-set, given its completeness, is on what scale the phenomenon of HI ablation holds. The H I deficiency parameter of individual galaxies is given in Fig. 4 as a function of the projected linear separation from the X-ray center of the Coma cluster, out to $15 \mathrm{Mpc}$, along with average values taken in bins of $0.5^{\circ}$ (from $0^{\circ}$ to $2^{\circ}$ ) and in bins of $1^{\circ}$ further out (see also Table 2). It is apparent that significant H I deficiency occurs out to approximately $3 \mathrm{Mpc}$ radius. At one virial radius (i.e. at 2.2 Mpc, Girardi et al. 1998; or 2.9 Mpc Lokas \& Mamon 2003; Neumann et al. 2001), the average H I content of

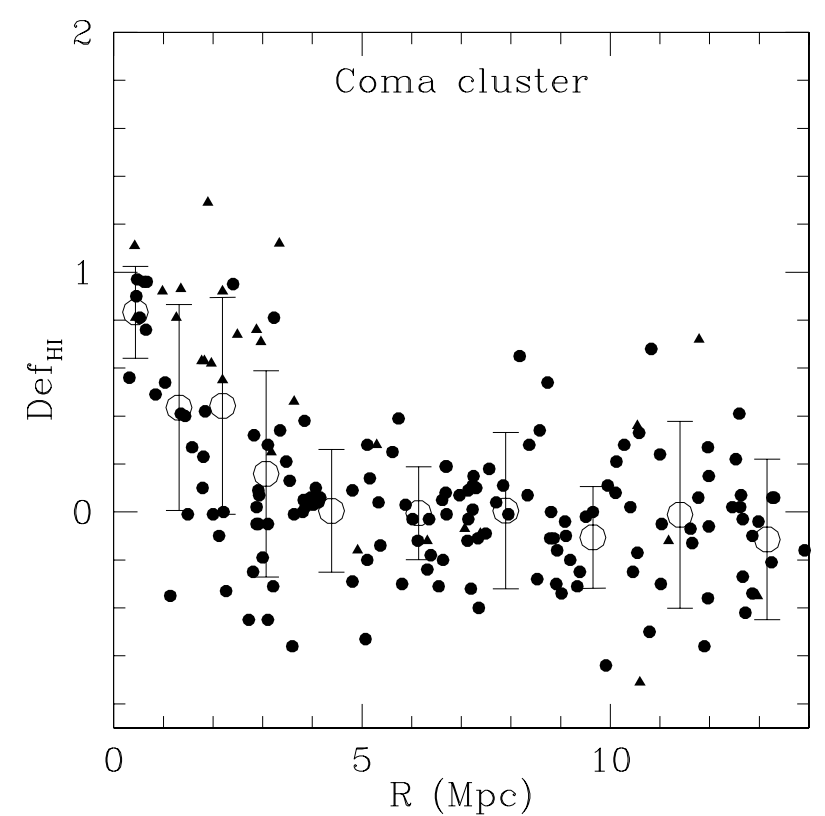

Fig. 4. The projected distribution about the X-ray center of the Coma cluster of the H I deficiency of Late-type Supercluster detected (dots) and undetected (triangles) members (excluding HL). Large circles represent averages in bins of $0.5^{\circ}\left(1^{\circ}\right)$. One $\sigma$ errorbars are given.

Table 2. The radial distribution of the mean binned H I deficiency of Late-type Supercluster members (excluding HL) about the Coma cluster.

\begin{tabular}{ccc}
\hline \hline$\Theta$ (Deg.) & $R(\mathrm{Mpc})$ & $\left\langle\right.$ Def $\left._{\mathrm{HI}}\right\rangle$ \\
\hline $0.0-0.5$ & 0.42 & $0.83 \pm 0.19$ \\
$0.5-1.0$ & 1.25 & $0.43 \pm 0.43$ \\
$1.0-1.5$ & 2.09 & $0.44 \pm 0.45$ \\
$1.5-2.0$ & 2.93 & $0.16 \pm 0.43$ \\
$2.0-3.0$ & 4.19 & $0.00 \pm 0.25$ \\
$3.0-4.0$ & 5.87 & $0.00 \pm 0.19$ \\
$4.0-5.0$ & 7.55 & $0.00 \pm 0.32$ \\
$5.0-6.0$ & 9.24 & $-0.10 \pm 0.21$ \\
$6.0-7.0$ & 10.9 & $-0.01 \pm 0.39$ \\
$7.0-8.0$ & 12.6 & $-0.11 \pm 0.33$ \\
\hline
\end{tabular}

the supercluster galaxies becomes indistinguishable from that of the field, in agreement with Solanes et al. (2001) ${ }^{1}$.

The star-formation rate, as derived from $\mathrm{H} \alpha$ observations, shows a significant cut-off near 1 to 2 projected virial radii from the Coma cluster (Gavazzi et al. 2005b) and from overdensity peaks in the SDSS (Nichol 2004; Miller 2004). What the H I observations show is the driver of the SFR decline, i.e. the lack of gas to sustain it.

${ }^{1}$ Galaxies in the HL, identified as such outward of $2^{\circ}$ from Coma and A1367, on average do not show significant deficiency are not included in Fig. 4. 
Table 3. Parameters of the newly observed galaxies.

\begin{tabular}{|c|c|c|c|c|c|c|c|c|c|c|}
\hline \multirow[t]{2}{*}{ Obj. } & RA & Dec & $V_{\text {opt }}$ & $\sigma$ & $S_{\mathrm{p}}$ & $V_{\mathrm{HI}}$ & $W_{50}$ & $W_{20}$ & $I_{\mathrm{HI}}$ & Qual. \\
\hline & \multicolumn{2}{|c|}{$\mathrm{J} 2000.0$} & $\mathrm{~km} \mathrm{~s}^{-1}$ & mJy/beam & $\mathrm{mJy} /$ beam & $\mathrm{km} \mathrm{s}^{-1}$ & $\mathrm{~km} \mathrm{~s}^{-1}$ & $\mathrm{~km} \mathrm{~s}^{-1}$ & $\mathrm{Jy} \mathrm{km} \mathrm{s}^{-1}$ & \\
\hline \multicolumn{11}{|l|}{ Coma Supercluster } \\
\hline CGCG 97-078 & 114316.24 & 194455.6 & $7560 \pm 70$ & 0.54 & - & - & - & - & - & - \\
\hline CGCG 127-018 & 113944.62 & 224107.7 & - & 0.56 & 17.0 & $6922 \pm 2$ & 147 & 168 & $2.09 \pm 0.06$ & 1 \\
\hline CGCG 127-039 & 114330.88 & 230043.3 & $6908 \pm 60$ & 0.62 & 22.4 & $6911 \pm 1$ & 37 & 58 & $0.82 \pm 0.04$ & 2 \\
\hline CGCG 97-124 & 114456.97 & 194353.9 & $7771 \pm 60$ & 0.66 & - & - & - & - & - & - \\
\hline CGCG 127-055 & 114646.66 & 211616.9 & $6615 \pm 52$ & 0.67 & 9.3 & $6626 \pm 3$ & 215 & 242 & $1.86 \pm 0.08$ & 1 \\
\hline CGCG 127-067 & 115039.40 & 205426.1 & $6349 \pm 39$ & 0.43 & 3.8 & $6400 \pm 16$ & 283 & 304 & $0.73 \pm 0.10$ & 1 \\
\hline CGCG 157-044 & 115123.19 & 264703.6 & $6624 \pm 44$ & 0.64 & 5.1 & $6607 \pm 4$ & 240 & 260 & $0.84 \pm 0.08$ & 1 \\
\hline CGCG 97-172 & 115214.30 & 183905.7 & $7650 \pm 56$ & 0.36 & 1.6 & $7826 \pm 16$ & 214 & 227 & $0.21 \pm 0.10$ & 3 \\
\hline CGCG 127-137W & 120141.90 & 202417.3 & 6794 & 0.49 & 3.4 & $6871 \pm 16$ & 357 & 398 & $1.07 \pm 0.10$ & 1 \\
\hline CGCG 98-023 & 120144.40 & 175353.8 & $6905 \pm 48$ & 0.38 & 5.8 & $6905 \pm 16$ & 262 & 282 & $1.01 \pm 0.10$ & 1 \\
\hline CGCG 127-136 & 120147.40 & 210506.7 & 6930 & 0.29 & 17.5 & $6675 \pm 16$ & 173 & 228 & $2.36 \pm 0.10$ & 2 \\
\hline CGCG 127-138 & 120155.47 & 204452.1 & $7192 \pm 46$ & 0.60 & - & - & - & - & - & - \\
\hline CGCG 128-015 & 120456.20 & 211427.5 & $6729 \pm 16$ & 0.44 & 19.0 & $6741 \pm 16$ & 102 & 124 & $1.64 \pm 0.10$ & 2 \\
\hline CGCG 99-002 & 121755.86 & 182357.8 & $7640 \pm 59$ & 0.90 & 2.7 & (7605) & - & - & - & 4 \\
\hline CGCG 128-072 & 121808.27 & 244118.5 & $6838 \pm 51$ & 0.63 & 8.3 & $6795 \pm 6$ & 119 & 174 & $0.82 \pm 0.07$ & 1 \\
\hline CGCG 99-013 & 121910.00 & 191626.9 & $7297 \pm 16$ & 0.36 & 7.2 & $7336 \pm 16$ & 235 & 251 & $1.41 \pm 0.10$ & 1 \\
\hline CGCG 128-081 & 122052.50 & 252547.2 & 7112 & 0.42 & 5.0 & $7204 \pm 16$ & 290 & 312 & $1.00 \pm 0.10$ & 1 \\
\hline CGCG 129-004 & 122949.40 & 222219.4 & $6847 \pm 70$ & 0.57 & 5.5 & $6736 \pm 7$ & 275 & 317 & $1.09 \pm 0.08$ & 1 \\
\hline CGCG 159-048 & 124011.35 & 311038.1 & $7096 \pm 44$ & 0.65 & 7.0 & $7064 \pm 8$ & 309 & 363 & $1.60 \pm 0.10$ & 1 \\
\hline CGCG 159-071 & 124543.41 & 292558.5 & $6936 \pm 44$ & 1.06 & 17.1 & $6971 \pm 1$ & 189 & 202 & $2.60 \pm 0.10$ & 1 \\
\hline CGCG 159-097 & 125206.77 & 270134.3 & $6573 \pm 190$ & 0.65 & 3.2 & $6424 \pm 30$ & 260 & 357 & $0.60 \pm 0.10$ & 3 \\
\hline CGCG 160-009 & 125432.95 & 282234.7 & $7079 \pm 63$ & 0.70 & - & - & - & - & - & - \\
\hline FOCA 636 & 125756.70 & 275930.0 & 4649 & 0.40 & 1.8 & $4605 \pm 30$ & 130 & 219 & $0.18 \pm 0.05$ & 3 \\
\hline FOCA 610 & 125757.70 & 280342.0 & 8299 & 0.38 & 1.7 & $8125 \pm 8$ & 211 & 236 & $0.19 \pm 0.05$ & 3 \\
\hline CGCG 130-003 & 125947.24 & 214845.7 & $7094 \pm 32$ & 0.55 & 4.4 & $7140 \pm 4$ & 335 & 357 & $0.51 \pm 0.08$ & 3 \\
\hline CGCG 160-261 & 130059.10 & 275358.6 & $6896 \pm 7$ & 0.37 & - & - & - & - & - & - \\
\hline CGCG 160-128 & 130422.57 & 284838.5 & $8054 \pm 36$ & 0.74 & 25.7 & $7920 \pm 1$ & 115 & 137 & $2.46 \pm 0.07$ & 1 \\
\hline CGCG 160-138 & 130635.52 & 271006.2 & $7852 \pm 60$ & 0.37 & - & - & - & - & - & - \\
\hline CGCG 160-146 & 130814.00 & 273055.3 & $7337 \pm 23$ & 0.25 & - & - & - & - & - & - \\
\hline CGCG 160-169 & 131440.60 & 295951.8 & $6960 \pm 60$ & 0.69 & 6.2 & $6850 \pm 4$ & 298 & 318 & $1.26 \pm 0.10$ & 1 \\
\hline CGCG 130-029 & 131730.80 & 203555.1 & $6671 \pm 71$ & 0.36 & 4.6 & $6560 \pm 16$ & 317 & 325 & $1.30 \pm 0.10$ & 1 \\
\hline CGCG 160-195 & 131947.60 & 304931.9 & $7297 \pm 15$ & 0.34 & 5.0 & $7247 \pm 16$ & 158 & 223 & $0.63 \pm 0.10$ & 2 \\
\hline CGCG 161-048 & 132557.14 & 313703.6 & $7268 \pm 44$ & 0.66 & - & - & - & - & - & - \\
\hline CGCG 161-051 & 132643.29 & 303026.7 & $7150 \pm 60$ & 1.52 & - & - & - & - & - & - \\
\hline CGCG 161-054 & 132703.07 & 305834.3 & $7668 \pm 44$ & 0.62 & 7.0 & $6756 \pm 3$ & 284 & 303 & $1.34 \pm 0.09$ & 1 \\
\hline \multicolumn{11}{|l|}{ Virgo Cluster } \\
\hline VCC 30 & 121054.50 & 155654.6 & - & 0.92 & 9.5 & $2084 \pm 16$ & 116 & 148 & $0.83 \pm 0.10$ & 2 \\
\hline VCC 85 & 121336.50 & 130201.1 & - & 0.32 & 2.4 & $1932 \pm 16$ & 74 & 84 & $0.14 \pm 0.10$ & 2 \\
\hline VCC 113 & 121432.90 & 120612.0 & 2155 & 0.33 & 17.5 & $2091 \pm 16$ & 148 & 171 & $2.12 \pm 0.10$ & 2 \\
\hline VCC 137 & 121508.60 & 145819.5 & - & 0.56 & - & (4000) & - & - & - & 4 \\
\hline VCC 429 & 122044.20 & 143803.3 & - & 0.39 & 4.0 & $600 \pm 16$ & 87 & 109 & $0.27 \pm 0.10$ & 2 \\
\hline VCC 578 & 122243.60 & 183252.1 & - & 0.39 & 4.0 & $6490 \pm 16$ & 161 & 178 & $0.48 \pm 0.10$ & 1 \\
\hline VCC 1574 & 123432.90 & 151052.3 & - & 0.17 & 2.3 & $639 \pm 16$ & 127 & 161 & $0.22 \pm 0.10$ & 2 \\
\hline VCC 1623 & 123531.90 & 163646.9 & - & 0.30 & 1.9 & $2108 \pm 16$ & 78 & 91 & $0.13 \pm 0.10$ & 2 \\
\hline VCC 1821 & 124008.90 & 065302.1 & - & 0.31 & 2.5 & $1007 \pm 16$ & 75 & 87 & $0.16 \pm 0.10$ & 2 \\
\hline VCC 1873 & 124118.60 & 063127.0 & - & 0.26 & 17.0 & $1692 \pm 16$ & 112 & 134 & $1.37 \pm 0.10$ & 2 \\
\hline VCC 1898 & 124157.50 & 034909.4 & $881 \pm 23$ & 0.50 & - & - & - & - & - & - \\
\hline VCC 2071 & 124825.40 & 091856.8 & - & 0.32 & 2.7 & $6484 \pm 16$ & 185 & 196 & $0.40 \pm 0.10$ & 2 \\
\hline CGCG 43066 & 125515.40 & 025348.4 & $2798 \pm 23$ & 0.47 & 21.0 & $2802 \pm 16$ & 382 & 403 & $6.09 \pm 0.10$ & 1 \\
\hline
\end{tabular}




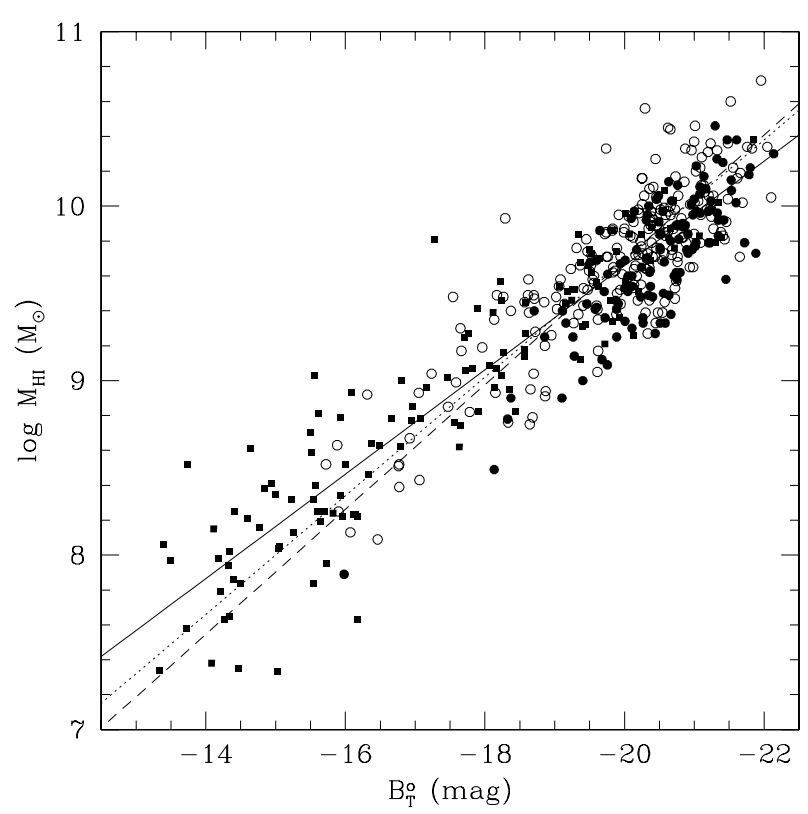

Fig. 5. The correlation between $\log M_{\mathrm{HI}}$ and $B_{\mathrm{T}}^{\circ}$ for $\mathrm{Sa}-\mathrm{Sb}$ (filled circles), Sbc-Sc (empty circles), Scd-Im-BCD (filled squares). The direct fit (solid line), the inverse fit (dashed) (obtained considering all Hubble types), and the relation used in Paper I (dotted) are given.

\subsection{The HI mass $-B_{T}^{\circ}$ relation}

Taking advantage of the large sample of optically selected galaxies with H I measurements and optical ( $B$-band) photometry (accurate to within $0.1 \mathrm{mag}$ ), we study the HI mass vs. $B_{\mathrm{T}}^{\circ}$ relation for unperturbed galaxies. However, since the Coma Supercluster sample $\left(m_{\mathrm{p}} \leq 15.7\right)$ contains only giant galaxies brighter than $M_{\mathrm{p}}=-19.1$, we have included the Virgo galaxies (Paper I) for the purpose of extending this correlation over a broader magnitude range. In doing so we must obviously exclude galaxies with perturbed $\mathrm{H}$ I contents. Conservatively, we excluded galaxies with $\operatorname{Def}_{\mathrm{HI}} \geq 0.2$ (the $\operatorname{Def}_{\mathrm{HI}}$ parameter is determined from diameters (Sect. 5.1), independent of the $B$ luminosity, as in Haynes \& Giovanelli 1984). Figure 5 shows the relation for the 465 late-type galaxies that were selected accordingly, and plotted in three bins of Hubble type to stress the consistency among them. The three given linear regressions, obtained combining all Hubble types, are the direct one $\left(M_{\mathrm{HI}}=\right.$ $\left.3.680-0.299 \times M_{\mathrm{p}}\right)$, the inverse one $\left(M_{\mathrm{p}}=7.090-2.795 \times M_{\mathrm{HI}}\right)$, and the one adopted in Paper I $\left(M_{\mathrm{HI}}=2.9-0.34 \times M_{\mathrm{p}}\right)$. The residual of the (direct) correlation is $\sigma\left(\log M_{\mathrm{HI}}\right)=0.26$; i.e., the $\mathrm{H}$ I mass of disk galaxies can be predicted within a factor of 1.8 uncertainty from their $B$ luminosity.

\subsection{The HI mass function}

Figure 6 shows the frequency distribution of $M_{\mathrm{HI}}$ for the cluster (Coma + A1367) late-type galaxies (solid histogram) and for the isolated galaxies (including HL), the latter normalized to the former by the ratio of the number of galaxies. The H I Mass Function (HIMF) so obtained cannot be meaningfully compared with the one of Virgo, obtained in Paper I, or even with the one of isolated galaxies by Zwaan et al. (2003),

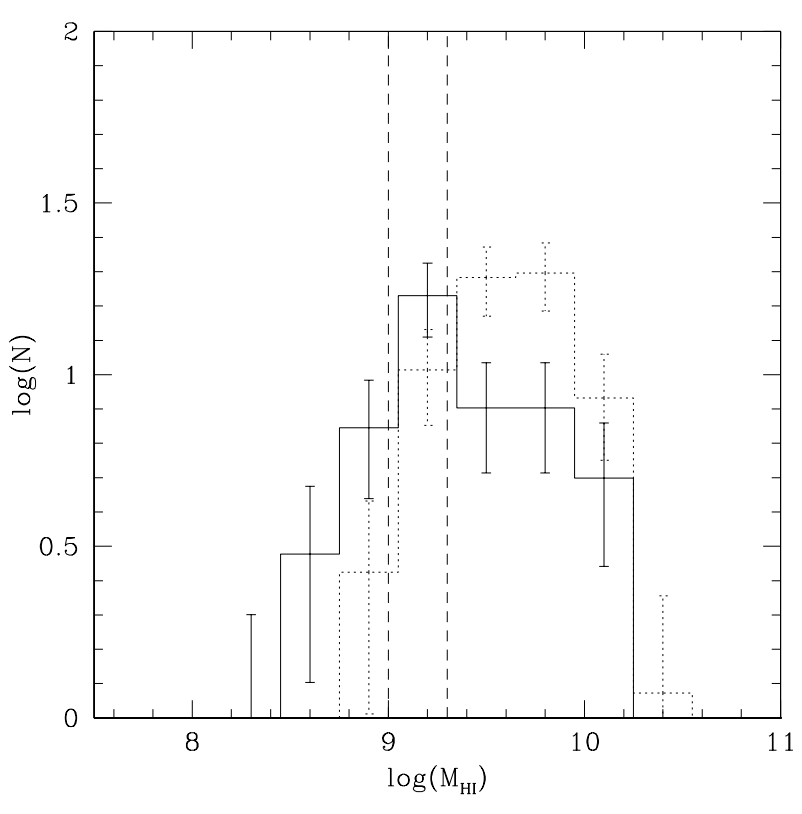

Fig. 6. The MHI function for the members of the Coma+A1367 clusters (solid) and for the non-cluster Supercluster members (dotted). The optical $\left(\log M_{\mathrm{HI}}=9.3 M_{\odot}\right)$ and radio $\left(\log M_{\mathrm{HI}}=9.0 M_{\odot}\right)$ completeness limits are drawn.

because of the shallowness of the optical and $21 \mathrm{~cm}$ observations available for the Coma Supercluster. Given the relation $M_{\mathrm{HI}}=3.680-0.299 \times M_{\mathrm{p}}$, discussed in the previous section, the limiting magnitude $m_{\mathrm{p}} \leq 15.7\left(M_{\mathrm{p}} \geq-19.1\right)$ of the CGCG implies that, on average, only galaxies with $\log M_{\mathrm{HI}}>9.3 M_{\odot}$ are targeted. This imposes a completeness cut-off that is even shallower than the limiting detectable $\mathrm{HI}$ mass of $\log M_{\mathrm{HI}}>$ $9.0 M_{\odot}$ that derives from the typical noise figure of the $21 \mathrm{~cm}$ observations $(\langle\sigma\rangle \sim 1 \mathrm{mJy})$. In Paper I we showed that significant differences between the HIMF of the Virgo cluster and of the field occur for $\log M_{\mathrm{HI}}<9.0 M_{\odot}$, i.e. below both the present cut-off lines. For $9.5<\log M_{\mathrm{HI}}<10 M_{\odot}$, however, there is in Fig. 6 a barely significant excess in the frequency of non-cluster members of the Coma Supercluster with respect to cluster members. This can be understood as a signature of the deficiency pattern of cluster galaxies, as found in Paper I for Virgo.

\section{Summary and conclusions}

We observed 35 galaxies in the Coma Supercluster and 13 in the Virgo cluster in the 21-cm H I line with the refurbished Arecibo telescope. The high sensitivity of our observations (rms noise $\sim 0.5 \mathrm{mJy}$ ) resulted in the detection of $37 \mathrm{ob}-$ jects, and significant upper limits were obtained for the remaining ones. Including the present observations, the H I survey of the Coma Supercluster has reached virtual completion (94\% among the late-type members). And, by combining all data, we have determined with high significance that the typical scale of $\mathrm{H}$ I deficiency around the Coma cluster is $2-3 \mathrm{Mpc}$, i.e. one virial radius.

With the present data, a meaningful determination of the HIMF can be only obtained for $\log M_{\mathrm{HI}}>9.0 M_{\odot}$, insufficient for comparing it with the deeper HIMF of Virgo (Paper I) 
and of isolated galaxies (Zwaan et al. 2003). Comparing cluster with non-cluster Supercluster members, we do, however, detect a shortage of high H I mass galaxies among cluster members which can be attributed to the pattern of $\mathrm{HI}$ deficiency found in rich clusters.

Acknowledgements. The Arecibo Observatory is part of the National Astronomy and Ionosphere Center, which is operated by Cornell University under a cooperative agreement with the National Science Foundation. This research also made use of the Lyon-Meudon Extragalactic Database (LEDA), recently incorporated in HyperLeda and the NASA/IPAC Extragalactic Database (NED), operated by the Jet Propulsion Laboratory, California Institute of Technology, under contract with the National Aeronautics and Space Administration and the Goldmine database.

\section{References}

Beijersbergen, M. 2003, Ph.D. Thesis, Groningen University

Binggeli, B., Sandage, A., \& Tammann, G. A. 1985, AJ, 90, 1681 (VCC)

Bothun, G., Aaronson, M., Schommer, R., Mould, J., Huchra, J., \& Sullivan, W. 1985, ApJS, 57, 423

Bravo-Alfaro, H., Cayatte, V., van Gorkom, J. H., \& Balkowski, C. 2000, AJ, 119, 580

Bravo-Alfaro, H., Cayatte, V., van Gorkom, J. H., \& Balkowski, C. 2001, A\&A, 379, 347

Byrd, G., \& Valtonen, M. 1990, ApJ, 350, 89

Chincarini, G., Giovanelli, R., \& Haynes, M. P. 1983a, ApJ, 269, 13

Chincarini, G., Giovanelli, R., Haynes, M., \& Fontanelli, P. 1983b, ApJ, 267, 511

Cowie, L. L., \& Songaila, A. 1977, Nature, 266, 501

de Lapparent, V., Geller, M. J., \& Huchra, J. P. 1986, ApJ, 302, L1

Dell'Antonio, I., Bothun, G., \& Geller, M. 1996, AJ, 112, 1759

Dickey, J. M., \& Gavazzi, G. 1991, ApJ, 373, 347

Eder, J., Giovanelli, R., \& Haynes, M. 1991, AJ, 102, 572

Fontanelli, P. 1984, A\&A, 138, 85

Gavazzi, G. 1987, ApJ, 320, 96

Gavazzi, G. 1989, ApJ, 346, 59

Gavazzi, G., Carrasco, L., \& Galli, R. 1999, A\&AS, 136, 227

Gavazzi, G., Boselli, A., Donati, A., Franzetti, P., \& Scodeggio, M. 2003, A\&A, 400, 451

Gavazzi, G., Boselli, A., van Driel, W., \& O’Neil, K. 2005a, A\&A, 429, 439 (Paper I)

Gavazzi, G., Boselli, Cortese, L., Arosio, I., et al. 2005b, A\&A, submitted
Giovanelli, R., \& Haynes, M. 1985, ApJ, 292, 404

Giovanelli, R. 2005, AJ, in press

Girardi, M., Giuricin, G., Mardirossian, F., Mezzetti, M., \& Boschin, W. 1998, ApJ, 505, 74

Gunn, J. E., \& Gott, J. R. III 1972, ApJ, 176, 1

Haynes, M., \& Giovanelli, R. 1984, AJ, 89, 758

Haynes, M., Giovanelli, R., \& Chincarini, G. 1984, ARA\&A, 22, 445

Haynes, M., Giovanelli, R., Herter, T., et al. 1997, AJ, 113, 1197

Lewis, B., Helou, G., \& Salpeter, E. 1985, ApJS, 59, 161

Lokas, E. L., \& Mamon, G. A. 2003, MNRAS, 343, 401

Lu, N., Hoffman, G. L., Groff, T., Ross, T., \& Lamphier, C., 1993, ApJS, 88, 383

Magri, C. 1994, AJ, 108, 896

Miller, C. J. 2004, in Clusters of Galaxies: Probes of Cosmological Structure and Galaxy Evolution, from the Carnegie Observatories Centennial Symposia (Cambridge University Press), as part of the Carnegie Observatories Astrophysics Series, ed. J. S. Mulchaey, A. Dressler, \& A. Oemler

Moore, B., Katz, N., Lake, G., Dressler, A., \& Oemler, A., Jr. 1996, Nature, 379, 613

Mould, J., Martin, S., Bothum, G., Huchra, J., \& Shomma, B. 1995, ApJS, 96, 1

Neumann, D. M., Arnaud, M., Gastaud, R., et al. 2001, A\&A, 365, L74

Nichol, R. C. 2004, in Clusters of Galaxies: Probes of Cosmological Structure and Galaxy Evolution, from the Carnegie Observatories Centennial Symposia (Cambridge University Press), part of the Carnegie Observatories Astrophysics Series, ed. J. S. Mulchaey, A. Dressler, \& A. Oemler, 24

Nulsen, P. E. J. 1982, MNRAS, 198, 1007

O'Neil, K. 2004, AJ, 128, 2080

Ramella, M., Geller, M. J., \& Huchra, J. P. 1992, ApJ, 384, 396

Salzer, J., Hanson, M., \& Gavazzi, G. 1990, ApJ, 353, 39

Scodeggio, M., \& Gavazzi, G. 1993, ApJ, 409, 110

Solanes, J., Manrique, A., Garcia-Gómez, C., et al. 2001, ApJ, 548, 97

Sulentic, J., \& Arp, H. 1982, AJ, 88, 489

Sullivan III, W. T., Bothun, G. D., Bates, B., \& Schommer, R. A. 1981, AJ, 86, 919

van Driel, W., Ragaigne, D., Boselli, A., Donas, J., \& Gavazzi, G. 2000, A\&AS, 144, 463

Vogt, N. P., Haynes, M. P., Herter, T., \& Giovanelli, R. 2004, AJ, 127, 3273

Williams, B., \& Kerr, F. 1981, AJ, 86, 953

Zwaan, M. A., Staveley-Smith, L., Korivalski, B., et al. 2003, AJ, 125, 2842 


\section{Online Material}


G. Gavazzi et al.: H I observations in the Coma Supercluster, Online Material p 2

Table 4. Basic H I properties of late-type members to the Coma Supercluster (including HL).

\begin{tabular}{|c|c|c|c|c|c|c|c|c|}
\hline CGCG & Type & $\begin{array}{l}m_{\mathrm{p}} \\
\mathrm{mag}\end{array}$ & Cloud & $\begin{array}{c}V \\
\mathrm{~km} \mathrm{~s}^{-1}\end{array}$ & $\begin{array}{r}\log M_{\mathrm{HI}} \\
M_{\odot}\end{array}$ & Ref. & $\operatorname{Def}_{\mathrm{HI}}$ & Qual \\
\hline 097-005 & Sc & 15.5 & Isol. & 6129 & 9.72 & 126 & -0.29 & 1 \\
\hline 097-026 & Pec & 13.9 & Pair & 6202 & 9.88 & 39 & -0.45 & 1 \\
\hline 097-027 & $\mathrm{Sc}$ & 14.6 & Pair & 6630 & 9.27 & 24 & 0.28 & 2 \\
\hline 097-036 & S.. & 15.7 & Pair & 6595 & - & - & - & - \\
\hline 097-062 & Pec & 15.5 & A1367 & 7809 & 9.33 & 40 & 0.31 & 1 \\
\hline 097-063 & Pec & 15.7 & A1367 & 6102 & 9.09 & 24 & 0.22 & 2 \\
\hline 097-064 & S.. & 15.6 & A1367 & 5976 & 9.17 & 126 & 0.03 & 3 \\
\hline 097-068 & Sbc & 14.7 & A1367 & 5974 & 9.99 & 126 & -0.14 & 1 \\
\hline 097-072 & $\mathrm{Sa}$ & 15.0 & A1367 & 6332 & 9.14 & 126 & 0.50 & 1 \\
\hline 097-073 & Pec & 15.6 & A1367 & 7290 & 9.31 & 40 & 0.16 & 2 \\
\hline 097-076 & $\mathrm{Sb}$ & 15.5 & A1367 & 7060 & $<8.39$ & 40 & $>1.39$ & - \\
\hline 097-078 & $\mathrm{Sa}$ & 15.2 & A1367 & 7526 & $<8.68$ & 193 & $>1.28$ & - \\
\hline 097-079 & Pec & 15.7 & A1367 & 6996 & 9.21 & 40 & 0.25 & 1 \\
\hline 097-082 & $\mathrm{Sa}$ & 15.0 & A1367 & 6100 & $<8.68$ & 2 & $>1.01$ & 5 \\
\hline 097-087 & Pec & 14.3 & A1367 & 6735 & 9.83 & 40 & 0.19 & 1 \\
\hline 097-091 & $\mathrm{Sa}$ & 14.7 & A1367 & 7368 & 9.77 & 126 & -0.18 & 1 \\
\hline 097-092 & $\mathrm{Sbc}$ & 15.5 & A1367 & 6373 & 9.18 & 24 & 0.31 & 4 \\
\hline 097-093 & Pec & 15.5 & A1367 & 4857 & 9.03 & 4 & 0.57 & 4 \\
\hline $097-102 \mathrm{~N}$ & $\mathrm{Sa}$ & 15.1 & A1367 & 6361 & 9.21 & 40 & 0.36 & 1 \\
\hline 097-111S & Pec & 16.5 & A1367 & 7239 & - & - & - & - \\
\hline 097-114 & Pec & 15.4 & A1367 & 8257 & 9.44 & 193 & -0.17 & 1 \\
\hline 097-119 & $\mathrm{Sa}$ & 15.7 & A1367 & 5256 & 8.92 & 4 & 0.22 & 1 \\
\hline 097-120 & $\mathrm{Sa}$ & 14.5 & A1367 & 5595 & 8.80 & 4 & 0.90 & 4 \\
\hline 097-121 & Sab & 14.6 & A1367 & 6571 & 9.37 & 126 & 0.28 & 1 \\
\hline 097-122 & Pec & 14.9 & A1367 & 5468 & 9.35 & 126 & 0.49 & 1 \\
\hline $097-129 \mathrm{E}$ & $\mathrm{Sbc}$ & 15.7 & A1367 & 6009 & - & - & - & - \\
\hline 097-129W & $\mathrm{Sb}$ & 14.0 & A1367 & 5082 & 10.09 & 24 & 0.18 & 1 \\
\hline 097-130 & $\mathrm{Sa}$ & 15.5 & A1367 & 6697 & $<8.83$ & 1 & $>0.40$ & 5 \\
\hline 097-138 & Pec & 15.5 & A1367 & 5317 & 9.69 & 40 & -0.22 & 3 \\
\hline 097-149 & S.. & 15.6 & A1367 & 6060 & $<9.25$ & 105 & $>0.07$ & - \\
\hline 097-151 & $\mathrm{Sab}$ & 15.6 & Isol. & 5854 & 9.05 & 88 & 0.34 & 1 \\
\hline 097-152 & $\mathrm{Sa}$ & 15.5 & A1367 & 6166 & 9.40 & 126 & 0.23 & 1 \\
\hline $097-168$ & S.. & 15.7 & Isol. & 5996 & $<8.91$ & 88 & $>0.25$ & - \\
\hline 097-169 & $\mathrm{Sc}$ & 15.7 & Pair & 5975 & 9.45 & 6 & 0.05 & 2 \\
\hline $097-172$ & S.. & 15.7 & Pair & 7826 & 8.71 & 193 & 0.38 & 3 \\
\hline 098-002 & $\mathrm{Sb}$ & 15.6 & Isol. & 6206 & 9.41 & 88 & 0.10 & 1 \\
\hline 098-007 & $\mathrm{Sbc}$ & 15.5 & Isol. & 6350 & 9.86 & 88 & -0.30 & 1 \\
\hline 098-013 & $\mathrm{Sc}$ & 15.1 & Isol. & 6949 & 9.65 & 40 & -0.12 & 1 \\
\hline 098-016 & $\mathrm{Sc}$ & 15.3 & Isol. & 6449 & 9.72 & 15 & 0.11 & 1 \\
\hline 098-017 & $\mathrm{Sbc}$ & 15.7 & Isol. & 7015 & 9.66 & 40 & 0.05 & 2 \\
\hline 098-023 & $\mathrm{Sb}$ & 15.1 & Tripl. & 6905 & 9.30 & 193 & -0.01 & 1 \\
\hline 098-034 & S.. & 14.8 & $\mathrm{~N} 4065 \mathrm{G}$ & 6432 & 9.07 & 40 & 0.11 & 3 \\
\hline 098-041 & $\mathrm{Sc}$ & 15.7 & $\mathrm{~N} 4065 \mathrm{G}$ & 7551 & 9.28 & 24 & 0.65 & 2 \\
\hline 098-046 & $\mathrm{Sa}$ & 14.3 & N4065 G & 6220 & 9.58 & 24 & 0.07 & 1 \\
\hline 098-050 & $\mathrm{Sc}$ & 14.1 & IC202 HL & 4372 & 9.44 & 24 & -0.04 & 1 \\
\hline 098-051 & $\mathrm{Sa}$ & 14.6 & IC202 HL & 4283 & 9.6 & 88 & -0.35 & 4 \\
\hline 098-058 & $\mathrm{Sbc}$ & 14.7 & Isol. & 7207 & 9.95 & 24 & 0.00 & 1 \\
\hline 098-067 & S.. & 15.7 & Isol. & 7610 & $<9.64$ & 184 & $>-0.71$ & - \\
\hline 098-071 & Pec & 15.5 & Pair & 6881 & 9.62 & 184 & -0.05 & 1 \\
\hline 098-072 & S.. & 15.7 & Pair & 7946 & - & - & - & - \\
\hline 098-073 & $\mathrm{Sb}$ & 15.7 & Tripl. & 6440 & - & - & - & - \\
\hline 098-074 & $\mathrm{Sa}$ & 15.6 & Isol. & 7471 & $<9.10$ & 88 & $>0.36$ & - \\
\hline 098-081 & $\mathrm{Sa}$ & 15.2 & Pair & 7177 & - & - & - & - \\
\hline 098-085 & Sc & 14.7 & Tripl. & 7042 & 9.69 & 24 & -0.13 & 2 \\
\hline 098-087 & S.. & 15.3 & Pair & 7540 & - & - & - & - \\
\hline 098-116 & $\mathrm{Sc}$ & 14.9 & Isol. & 6229 & 9.91 & 24 & -0.34 & 1 \\
\hline 099-002 & S.. & 15.5 & Isol. & 7605 & 9.02 & 193 & -0.08 & 3 \\
\hline 099-013 & $\mathrm{Sc}$ & 15.7 & Isol. & 7736 & 9.50 & 193 & -0.40 & 1 \\
\hline
\end{tabular}


G. Gavazzi et al.: H I observations in the Coma Supercluster, Online Material p 3

Table 4. continued.

\begin{tabular}{|c|c|c|c|c|c|c|c|c|}
\hline CGCG & Type & $\begin{array}{l}m_{\mathrm{p}} \\
\mathrm{mag}\end{array}$ & Cloud & $\begin{array}{c}V \\
\mathrm{~km} \mathrm{~s}^{-1}\end{array}$ & $\begin{array}{r}\log M_{\mathrm{HI}} \\
M_{\odot}\end{array}$ & Ref. & $\operatorname{Def}_{\mathrm{HI}}$ & Qual \\
\hline $100-005$ & Pec & 14.4 & Isol. & 6611 & 9.37 & 24 & 0.41 & 1 \\
\hline $100-012$ & Pec & 15.3 & Isol. & 6481 & 9.32 & 24 & -0.12 & 2 \\
\hline $101-033$ & $\mathrm{Sc}$ & 15.7 & Isol. & 6729 & 9.68 & 43 & 0.07 & 1 \\
\hline $101-043$ & $\mathrm{Sa}$ & 15.0 & Isol. & 6677 & $<8.74$ & 88 & $>0.96$ & - \\
\hline $101-049$ & Sbc & 14.9 & Isol. & 7148 & 10.06 & 7 & -0.24 & 2 \\
\hline $101-054$ & Sab & 13.8 & Isol. & 6606 & 9.92 & 24 & -0.20 & 3 \\
\hline $127-005$ & Sbc & 15.4 & Isol. & 6864 & 9.64 & 6 & 0.04 & 2 \\
\hline $127-018$ & $\mathrm{Sb}$ & 15.0 & Isol. & 6922 & 9.63 & 193 & -0.14 & 1 \\
\hline $127-025 \mathrm{~N}$ & $\mathrm{Sc}$ & 15.3 & Pair & 7142 & - & - & - & - \\
\hline $127-025 \mathrm{~S}$ & Sbc & 14.5 & Pair & 7076 & 9.87 & 126 & 0.09 & 1 \\
\hline $127-026$ & Sbc & 14.8 & Isol. & 6871 & 9.95 & 24 & -0.17 & 1 \\
\hline $127-033$ & $\mathrm{Sc}$ & 15.2 & Isol. & 6300 & 9.77 & 24 & 0.00 & 1 \\
\hline $127-035$ & $\mathrm{Sa}$ & 15.4 & Isol. & 6817 & 9.49 & 88 & 0.15 & 1 \\
\hline $127-037$ & $\mathrm{Pec}$ & 15.4 & Isol. & 6186 & 9.54 & 24 & -0.10 & 2 \\
\hline $127-038$ & $\mathrm{Sc}$ & 14.0 & Isol. & 6913 & 10.36 & 2 & -0.20 & 1 \\
\hline $127-039$ & Sbc & 15.3 & Isol. & 6911 & 9.21 & 193 & 0.25 & 2 \\
\hline $127-049$ & $\mathrm{Pec}$ & 15.5 & A1367 & 7061 & 9.34 & 6 & 0.32 & 1 \\
\hline $127-050$ & Sbc & 14.8 & N3937 G & 6752 & 9.95 & 2 & 0.00 & 1 \\
\hline $127-051 \mathrm{~N}$ & $\mathrm{Pec}$ & 15.8 & A1367 & 7288 & - & - & - & - \\
\hline $127-052$ & $\mathrm{Sa}$ & 14.0 & A1367 & 6946 & 9.73 & 126 & 0.16 & 1 \\
\hline $127-053$ & Sbc & 15.4 & Isol. & 6409 & $1-$ & 24 & -0.11 & 1 \\
\hline $127-054$ & $\mathrm{Sb}$ & 14.2 & N3937 G & 7026 & 10.38 & 2 & 0.10 & 1 \\
\hline $127-055$ & Pec & 15.1 & Isol. & 6626 & 9.54 & 193 & -0.45 & 1 \\
\hline $127-056$ & $\mathrm{Sb}$ & 15.7 & Isol. & 6814 & 9.67 & 126 & -0.05 & 1 \\
\hline $127-061$ & $\mathrm{Sc}$ & 15.4 & Isol. & 5954 & 9.85 & 24 & -0.20 & 1 \\
\hline $127-067$ & S.. & 15.5 & N3937 G & 6400 & 9.18 & 193 & -0.31 & 1 \\
\hline $127-071$ & $\mathrm{Pec}$ & 15.4 & N3937 G & 6388 & 9.36 & 24 & 0.13 & 2 \\
\hline $127-072$ & $\mathrm{Sc}$ & 14.6 & N3937 G & 6438 & 9.89 & 3 & -0.05 & 1 \\
\hline $127-073$ & $\mathrm{Sb}$ & 15.1 & N3937 G & 6439 & 9.01 & 1 & 0.81 & 4 \\
\hline $127-082$ & $\mathrm{Sc}$ & 14.7 & N3937 G & 6654 & 9.47 & 2 & 0.02 & 2 \\
\hline $127-083$ & Sbc & 15.1 & N3937 G & 6743 & 9.39 & 6 & 0.06 & 3 \\
\hline $127-085$ & $\mathrm{Sa}$ & 15.5 & N3937 G & 6595 & $<8.99$ & 88 & $>0.46$ & - \\
\hline $127-087$ & $\mathrm{Sbc}$ & 15.4 & N4005 HL & 4941 & 9.66 & 126 & 0.05 & 1 \\
\hline $127-095$ & $\mathrm{Sc}$ & 14.2 & N3937 G & 6199 & 10.04 & 2 & 0.03 & 1 \\
\hline 127-099 & $\mathrm{Sc}$ & 14.5 & Pair & 6458 & 9.71 & 126 & 0.10 & 1 \\
\hline $127-100$ & $\mathrm{Sb}$ & 14.9 & N3937 G & 6854 & 9.58 & 2 & 0.06 & 3 \\
\hline $127-104$ & $\mathrm{Sbc}$ & 15.5 & Isol. & 6814 & 9.63 & 6 & 0.19 & 1 \\
\hline $127-106$ & $\mathrm{Sb}$ & 14.5 & N4005 HL & 5027 & 9.88 & 24 & -0.32 & 1 \\
\hline $127-107$ & Sbc & 15.7 & Isol. & 6355 & 9.36 & 126 & 0.02 & 3 \\
\hline $127-109$ & $\mathrm{Sbc}$ & 15.7 & N4005 HL & 4731 & 9.4 & 32 & -0.04 & 2 \\
\hline $127-110$ & $\mathrm{Sbc}$ & 14.4 & N4005 HL & 4495 & 9.81 & 24 & 0.22 & 1 \\
\hline $127-111$ & Sbc & 15.7 & N4005 HL & 4617 & 9.47 & 111 & -0.41 & 1 \\
\hline $127-112$ & Sbc & 14.8 & N4005 HL & 4828 & 9.63 & 32 & 0.25 & 1 \\
\hline $127-114 \mathrm{E}$ & Pec & 15.0 & N4005 HL & 4771 & 9.84 & 24 & -0.02 & 3 \\
\hline $127-114 \mathrm{~W}$ & Pec & 15.0 & N4005 HL & 4771 & 9.84 & 24 & -0.13 & 3 \\
\hline $127-118$ & $\mathrm{Sc}$ & 15.2 & N4005 HL & 4551 & 9.38 & 24 & 0.13 & 1 \\
\hline $127-120$ & $\mathrm{Sb}$ & 14.1 & N4005 HL & 4470 & 9.32 & 24 & 0.22 & 3 \\
\hline $127-121$ & $\mathrm{Sb}$ & 15.7 & N4005 HL & 4131 & - & - & - & - \\
\hline $127-123$ & $\mathrm{Sc}$ & 14.7 & N4005 HL & 4479 & 9.84 & 24 & -0.03 & 1 \\
\hline $127-127$ & $\mathrm{Sb}$ & 14.6 & N4005 HL & 4048 & 9.34 & 111 & 0.14 & 1 \\
\hline $127-133$ & $\mathrm{Sc}$ & 15.3 & N4005 HL & 4667 & 9.12 & 32 & -0.08 & 1 \\
\hline $127-136$ & S.. & 15.7 & Pair & 6675 & 9.68 & 193 & -1.02 & 2 \\
\hline $127-137 \mathrm{~W}$ & S.. & 16.0 & N4065 G & 6871 & 9.38 & 193 & -0.32 & 1 \\
\hline $127-138$ & S.. & 15.5 & Isol. & 7210 & $<8.77$ & 193 & $>-0.09$ & - \\
\hline $127-139$ & $\mathrm{Sa}$ & 15.5 & Isol. & 6713 & 9.29 & 24 & 0.34 & 3 \\
\hline $128-002$ & S.. & 15.7 & $\mathrm{~N} 4065 \mathrm{G}$ & 6780 & - & - & - & - \\
\hline $128-003$ & Pec & 14.6 & Isol. & 6435 & 9.69 & 2 & -0.11 & 2 \\
\hline $128-015$ & $\mathrm{Sb}$ & 15.3 & Pair & 6741 & 9.51 & 193 & -0.11 & 2 \\
\hline
\end{tabular}


G. Gavazzi et al.: H I observations in the Coma Supercluster, Online Material p 4

Table 4. continued.

\begin{tabular}{|c|c|c|c|c|c|c|c|c|}
\hline CGCG & Type & $\begin{array}{l}m_{\mathrm{p}} \\
\mathrm{mag}\end{array}$ & Cloud & $\begin{array}{c}V \\
\mathrm{~km} \mathrm{~s}^{-1}\end{array}$ & $\begin{array}{r}\log M_{\mathrm{HI}} \\
M_{\odot}\end{array}$ & Ref. & $\operatorname{Def}_{\mathrm{HI}}$ & Qual \\
\hline $128-016$ & S.. & 15.2 & Isol. & 6619 & 9.30 & 24 & -0.31 & 1 \\
\hline $128-021$ & $\mathrm{Sbc}$ & 15.4 & Isol. & 7064 & 9.83 & 24 & 0.02 & 1 \\
\hline $128-023$ & $\mathrm{Sa}$ & 14.4 & N4065 G & 6719 & 9.92 & 2 & -0.30 & 1 \\
\hline $128-029 E$ & S.. & 16.7 & IC762 G & 6634 & - & - & - & - \\
\hline $128-029 \mathrm{~W}$ & S.. & 16.2 & $\mathrm{IC} 762 \mathrm{G}$ & 7158 & 9.39 & 40 & -0.10 & 3 \\
\hline $128-031 \mathrm{~N}$ & S.. & 16.5 & Isol. & 6936 & - & - & - & - \\
\hline $128-037$ & $\mathrm{Sbc}$ & 14.8 & IC762 G & 7194 & 9.53 & 2 & 0.11 & 2 \\
\hline $128-042 \mathrm{~N}$ & S.. & 16.3 & Isol. & 7319 & 9.78 & 43 & -0.30 & 3 \\
\hline $128-044$ & S.. & 15.7 & Isol. & 6922 & 10.25 & 7 & -0.36 & 1 \\
\hline $128-049$ & $\mathrm{Sc}$ & 15.0 & Isol. & 6445 & 9.21 & 24 & 0.49 & 4 \\
\hline $128-052$ & $\mathrm{Sb}$ & 15.6 & Isol. & 6692 & 9.48 & 22 & 0.07 & 2 \\
\hline $128-053$ & $\mathrm{Sbc}$ & 15.6 & Isol. & 7307 & 10.06 & 40 & 0.01 & 1 \\
\hline $128-057$ & S.. & 15.6 & Isol. & 6998 & $<9.51$ & 184 & $>-0.35$ & - \\
\hline $128-058$ & S.. & 15.7 & $\mathrm{~N} 4213 \mathrm{G}$ & 6778 & 9.52 & 7 & -0.25 & 2 \\
\hline $128-059$ & $\mathrm{Sb}$ & 15.6 & $\mathrm{~N} 4213 \mathrm{G}$ & 6228 & 9.95 & 7 & -0.28 & 1 \\
\hline $128-063$ & $\mathrm{Sa}$ & 15.3 & Pair & 6747 & 9.97 & 24 & 0.06 & 1 \\
\hline $128-066$ & S.. & 15.1 & $\mathrm{~N} 4213 \mathrm{G}$ & 6526 & $<9.11$ & 88 & $>0.34$ & - \\
\hline $128-069$ & $\mathrm{Sbc}$ & 15.6 & $\mathrm{~N} 4213 \mathrm{G}$ & 7188 & 9.16 & 111 & 0.28 & 2 \\
\hline $128-072$ & Pec & 15.4 & Isol. & 6795 & 9.21 & 193 & 0.02 & 1 \\
\hline $128-073$ & $\mathrm{Sb}$ & 14.7 & Isol. & 6948 & 9.96 & 126 & 0.01 & 1 \\
\hline $128-075$ & $\mathrm{Sc}$ & 15.5 & Isol. & 6682 & 9.93 & 126 & 0.10 & 3 \\
\hline $128-079$ & Sc & 15.6 & Isol. & 6630 & 9.71 & 40 & -0.25 & 2 \\
\hline $128-080$ & $\mathrm{Sb}$ & 15.0 & Isol. & 7349 & 9.38 & 24 & 0.05 & 3 \\
\hline $128-081 W$ & S.. & 16.5 & Isol. & 7204 & 9.33 & 193 & -0.29 & 1 \\
\hline $128-082$ & $\mathrm{Sb}$ & 15.7 & Isol. & 6910 & $1-$ & 111 & -0.05 & 1 \\
\hline $128-087$ & $\mathrm{Sc}$ & 15.3 & Isol. & 6671 & 9.55 & 5 & 0.13 & 1 \\
\hline $128-089$ & $\mathrm{Sa}$ & 14.2 & Isol. & 6841 & 9.23 & 40 & 0.35 & 3 \\
\hline $128-090$ & $\mathrm{Sc}$ & 15.5 & Isol. & 6776 & 9.87 & 7 & -0.35 & 1 \\
\hline 129-004 & S.. & 15.2 & Isol. & 6736 & 9.33 & 193 & -0.08 & 1 \\
\hline $129-009$ & $\mathrm{Sa}$ & 15.3 & Pair & 6415 & 9.27 & 24 & 0.11 & 1 \\
\hline $129-013$ & S.. & 15.7 & Isol. & 6962 & 9.53 & 184 & -0.03 & 1 \\
\hline $129-016$ & S.. & 15.5 & N4615 HL & 4979 & - & - & - & - \\
\hline $129-018$ & $\mathrm{Sc}$ & 13.8 & N4615 HL & 4716 & 10.05 & 24 & -0.23 & 1 \\
\hline $129-020$ & $\mathrm{Sb}$ & 14.8 & Isol. & 6579 & 9.50 & 15 & 0.14 & 3 \\
\hline $129-021$ & S.. & 15.3 & Isol. & 6697 & 9.85 & 24 & -1.15 & 2 \\
\hline $129-022$ & Sab & 14.4 & Isol. & 6972 & 9.98 & 2 & -0.25 & 3 \\
\hline $129-023$ & S.. & 15.7 & Isol. & 6746 & 9.41 & 184 & -0.42 & 4 \\
\hline $129-025$ & $\mathrm{Sc}$ & 13.5 & N4615 HL & 4380 & 10.02 & 6 & 0.12 & 1 \\
\hline $130-002$ & S.. & 15.6 & Isol. & 6663 & 9.61 & 184 & -0.64 & 1 \\
\hline $130-003$ & $\mathrm{Sb}$ & 15.4 & Isol. & 7140 & 9.04 & 193 & 0.68 & 3 \\
\hline $130-005$ & $\mathrm{Sbc}$ & 15.5 & Isol. & 7058 & 9.30 & 127 & 0.21 & 1 \\
\hline $130-006$ & Sbc & 15.0 & Isol. & 6521 & 9.33 & 24 & 0.04 & 2 \\
\hline $130-008$ & $\mathrm{Sc}$ & 14.9 & Isol. & 7266 & 9.73 & 2 & -0.53 & 2 \\
\hline 130-009 & Sbc & 15.3 & Pair & 6335 & 9.93 & 24 & -0.03 & 1 \\
\hline $130-012$ & $\mathrm{Sbc}$ & 15.2 & Pair & 7131 & 9.97 & 24 & 0.19 & 1 \\
\hline $130-014$ & Sbc & 15.1 & Isol. & 7096 & 9.57 & 126 & 0.09 & 1 \\
\hline $130-021$ & $\mathrm{Sa}$ & 15.4 & Isol. & 7163 & 9.33 & 127 & 0.18 & 1 \\
\hline $130-025$ & $\mathrm{Sa}$ & 15.5 & Isol. & 7001 & 9.68 & 15 & 0.02 & 1 \\
\hline $130-026$ & $\mathrm{Sc}$ & 15.5 & Quadr. & 6870 & 9.85 & 24 & -0.03 & 1 \\
\hline $130-027$ & Sbc & 15.6 & Quadr. & 6834 & 9.94 & 40 & -0.13 & 1 \\
\hline $130-029$ & $\mathrm{Sc}$ & 15.4 & Pair & 6560 & 9.39 & 193 & 0.04 & 1 \\
\hline $131-008$ & $\mathrm{Sbc}$ & 15.6 & Isol. & 5972 & 9.76 & 88 & -0.04 & 1 \\
\hline 131-009 & $\mathrm{Sc}$ & 15.3 & Isol. & 7522 & 9.64 & 24 & -0.04 & 1 \\
\hline $157-012$ & $\mathrm{Sbc}$ & 15.1 & Isol. & 6814 & 9.73 & 40 & -0.19 & 1 \\
\hline $157-032$ & $\mathrm{Sa}$ & 15.2 & Isol. & 6811 & 9.16 & 24 & 0.64 & 4 \\
\hline $157-035$ & $\mathrm{Sb}$ & 13.7 & Isol. & 6281 & 10.18 & 24 & -0.05 & 1 \\
\hline $157-044$ & Pec & 15.4 & Isol. & 6607 & 9.19 & 193 & 0.22 & 1 \\
\hline $157-051$ & $\mathrm{Sc}$ & 15.3 & N4005 HL & 5151 & 9.65 & 24 & -0.04 & 2 \\
\hline
\end{tabular}


G. Gavazzi et al.: H I observations in the Coma Supercluster, Online Material p 5

Table 4. continued.

\begin{tabular}{|c|c|c|c|c|c|c|c|c|}
\hline CGCG & Type & $\begin{array}{l}m_{\mathrm{p}} \\
\mathrm{mag}\end{array}$ & Cloud & $\begin{array}{c}V \\
\mathrm{~km} \mathrm{~s}^{-1}\end{array}$ & $\begin{array}{r}\log M_{\mathrm{HI}} \\
M_{\odot}\end{array}$ & Ref. & $\operatorname{Def}_{\mathrm{HI}}$ & Qual \\
\hline $157-062$ & Pec & 15.5 & Isol. & 6882 & 9.68 & 5 & 0.01 & 2 \\
\hline $157-064$ & $\mathrm{Sb}$ & 14.8 & Isol. & 6407 & 9.84 & 40 & 0.02 & 2 \\
\hline $157-075$ & $\mathrm{Sc}$ & 15.7 & Isol. & 6694 & 9.54 & 7 & 0.06 & 2 \\
\hline $157-077$ & S.. & 15.4 & N4005 HL & 4100 & - & - & - & - \\
\hline $158-009$ & $\mathrm{Sb}$ & 14.0 & Pair & 7494 & 9.07 & 24 & 0.65 & 4 \\
\hline $158-010$ & Sbc & 15.2 & Pair & 7930 & 9.46 & 24 & 0.17 & 2 \\
\hline $158-029$ & S.. & 14.1 & N4169 HL & 3836 & 8.98 & 22 & 0.46 & 2 \\
\hline $158-030$ & $\mathrm{Sab}$ & 14.6 & N4169 HL & 3970 & 9.51 & 22 & -0.35 & 2 \\
\hline $158-031$ & $\mathrm{Sb}$ & 13.8 & N4169 HL & 3825 & 10.03 & 39 & -0.13 & 1 \\
\hline $158-036$ & $\mathrm{Sb}$ & 13.8 & Isol. & 6532 & 9.96 & 2 & -0.20 & 1 \\
\hline $158-038$ & Sab & 15.3 & Isol. & 6725 & 9.36 & 40 & 0.07 & 1 \\
\hline $158-042$ & S.. & 14.8 & N4169 HL & 3868 & 8.97 & 88 & 0.11 & 2 \\
\hline $158-046$ & S.. & 15.0 & N4169 HL & 3848 & - & - & - & - \\
\hline $158-047$ & $\mathrm{Sb}$ & 13.5 & N4169 HL & 3903 & 9.79 & 39 & 0.07 & 1 \\
\hline $158-053 \mathrm{~N}$ & $\mathrm{Sa}$ & 14.7 & Pair & 6599 & 9.79 & 2 & -0.02 & 1 \\
\hline $158-054$ & $\mathrm{Pec}$ & 14.6 & Isol. & 7685 & 9.76 & 2 & -0.13 & 3 \\
\hline $158-055$ & $\mathrm{Sb}$ & 15.3 & Isol. & 7650 & 9.53 & 24 & 0.46 & 4 \\
\hline $158-056$ & $\mathrm{Sa}$ & 15.5 & Tripl. & 8102 & 9.53 & 24 & 0.16 & 1 \\
\hline $158-061$ & $\mathrm{Sa}$ & 13.7 & N4169 HL & 3876 & $<8.24$ & 168 & 0.84 & - \\
\hline $158-070$ & $\mathrm{Sbc}$ & 15.3 & Quadr. & 7634 & 9.58 & 5 & 0.23 & 1 \\
\hline $158-081$ & $\mathrm{Pec}$ & 14.5 & Isol. & 6734 & 9.26 & 2 & -0.03 & 2 \\
\hline $158-091$ & $\mathrm{Sab}$ & 15.7 & IC3165 G & 7607 & 9.54 & 2 & -0.17 & 2 \\
\hline $158-102$ & S.. & 15.7 & N4615 HL & 4515 & 9.49 & 7 & 0.03 & 1 \\
\hline $158-105$ & $\mathrm{Sbc}$ & 15.1 & Isol. & 6824 & 9.98 & 24 & -0.16 & 1 \\
\hline $158-112$ & Sbc & 14.4 & Pair & 7165 & 9.73 & 2 & 0.41 & 4 \\
\hline $159-004$ & S.. & 15.7 & Pair & 7004 & 9.11 & 88 & 0.15 & 2 \\
\hline $159-005$ & Sbc & 14.7 & Pair & 6996 & 9.61 & 2 & 0.27 & 2 \\
\hline $159-008$ & $\mathrm{Sb}$ & 14.6 & Isol. & 7393 & 10.03 & 126 & 0.06 & 1 \\
\hline 159-009 & Sab & 14.1 & N4615 HL & 4551 & 10.14 & 24 & -0.52 & 1 \\
\hline $159-010$ & $\mathrm{Sb}$ & 15.7 & Isol. & 7009 & 9.62 & 5 & 0.24 & 1 \\
\hline $159-019$ & Sbc & 14.9 & N4615 HL & 4573 & 9.62 & 6 & -0.09 & 1 \\
\hline 159-031 & $\mathrm{Sa}$ & 15.3 & Pair & 7511 & 9.91 & 127 & -0.16 & 1 \\
\hline $159-033$ & $\mathrm{Sa}$ & 15.0 & Isol. & 7674 & 9.34 & 126 & 0.54 & 1 \\
\hline $159-037$ & Sab & 14.6 & Isol. & 7291 & 9.73 & 126 & -0.28 & 1 \\
\hline $159-040$ & $\mathrm{Sa}$ & 15.2 & Isol. & 7019 & 9.93 & 24 & -0.34 & 1 \\
\hline $159-048$ & S.. & 15.5 & Isol. & 7064 & 9.53 & 193 & -0.25 & 1 \\
\hline $159-049 \mathrm{~S}$ & S.. & 15.7 & Pair & 6330 & - & - & - & - \\
\hline $159-054$ & $\mathrm{Sc}$ & 15.5 & N4615 HL & 4759 & 9.41 & 88 & -0.01 & 1 \\
\hline $159-055$ & Sbc & 15.6 & Pair & 7737 & 10.01 & 24 & 0.01 & 1 \\
\hline $159-058$ & $\mathrm{Sa}$ & 15.5 & Isol. & 6797 & $<9.50$ & 184 & $>-0.07$ & - \\
\hline $159-059$ & Sab & 14.5 & Isol. & 7528 & 9.76 & 2 & -0.31 & 2 \\
\hline $159-060$ & $\mathrm{Pec}$ & 15.5 & Isol. & 7182 & 9.67 & 5 & 0.04 & 3 \\
\hline 159-061 & Sbc & 14.8 & Isol. & 6966 & 9.49 & 2 & 0.28 & 3 \\
\hline $159-064$ & S.. & 15.6 & Isol. & 7265 & $<9.64$ & 184 & $>-0.12$ & - \\
\hline $159-068$ & $\mathrm{Sa}$ & 15.7 & Isol. & 6313 & 9.25 & 184 & 0.03 & 4 \\
\hline $159-071$ & $\mathrm{Sc}$ & 15.5 & Isol. & 6971 & 9.72 & 193 & -0.03 & 1 \\
\hline $159-072 \mathrm{~N}$ & Pec & 14.8 & Pair & 6631 & 10.09 & 2 & -0.03 & 4 \\
\hline $159-072 S$ & Pec & 14.8 & Pair & 6590 & 10.03 & 2 & -0.12 & 4 \\
\hline $159-076$ & Sbc & 14.5 & Isol. & 6743 & 9.51 & 2 & 0.39 & 1 \\
\hline $159-080$ & $\mathrm{Sb}$ & 15.7 & Isol. & 6859 & 9.54 & 126 & 0.14 & 1 \\
\hline $159-081$ & $\mathrm{Sbc}$ & 15.5 & Pair & 8116 & 9.85 & 40 & -0.20 & 1 \\
\hline $159-082$ & $\mathrm{Sc}$ & 14.8 & Pair & 8078 & 10.01 & 126 & -0.18 & 1 \\
\hline $159-090$ & $\mathrm{Sc}$ & 15.5 & Tripl. & 8315 & 10.16 & 126 & -0.56 & 1 \\
\hline $159-091$ & S.. & 15.1 & Isol. & 6443 & 9.16 & 126 & 0.00 & 1 \\
\hline $159-092$ & $\mathrm{Sc}$ & 14.9 & N4615 HL & 4754 & 9.94 & 126 & 0.02 & 1 \\
\hline $159-093$ & $\mathrm{Sc}$ & 15.3 & N4615 HL & 5446 & $<8.58$ & 40 & $>0.63$ & 5 \\
\hline $159-095$ & Sbc & 14.9 & Isol. & 6837 & 9.64 & 2 & -0.24 & 1 \\
\hline $159-096$ & $\mathrm{Sc}$ & 15.1 & Isol. & 6186 & 9.96 & 126 & -0.01 & 1 \\
\hline
\end{tabular}


G. Gavazzi et al.: H I observations in the Coma Supercluster, Online Material p 6

Table 4. continued.

\begin{tabular}{|c|c|c|c|c|c|c|c|c|}
\hline CGCG & Type & $\begin{array}{l}m_{\mathrm{p}} \\
\mathrm{mag}\end{array}$ & Cloud & $\begin{array}{c}V \\
\mathrm{~km} \mathrm{~s}^{-1}\end{array}$ & $\begin{array}{r}\log M_{\mathrm{HI}} \\
M_{\odot}\end{array}$ & Ref. & $\operatorname{Def}_{\mathrm{HI}}$ & Qual \\
\hline $159-097$ & $\mathrm{Pec}$ & 15.4 & Isol. & 6424 & 9.02 & 193 & 0.21 & 3 \\
\hline $159-101$ & Pec & 15.3 & Coma & 7745 & 9.12 & 6 & 0.07 & 1 \\
\hline $159-102$ & $\mathrm{Sab}$ & 14.5 & Coma & 7061 & 9.97 & 126 & -0.25 & 3 \\
\hline $160-001$ & $\mathrm{Sb}$ & 15.6 & Coma & 7945 & 9.44 & 88 & 0.09 & 1 \\
\hline $160-005$ & $\mathrm{Sb}$ & 14.8 & Isol. & 6319 & 10.06 & 126 & -0.01 & 1 \\
\hline $160-007$ & S.. & 15.4 & Coma & 6462 & $<8.82$ & 88 & $>0.74$ & - \\
\hline $160-009$ & S.. & 15.5 & Coma & 7132 & $<8.84$ & 193 & $>0.55$ & - \\
\hline $160-012$ & S.. & 15.7 & Isol. & 6348 & $<9.36$ & 184 & $>-0.16$ & - \\
\hline $160-015$ & S.. & 15.5 & Coma & 7443 & $<8.90$ & 40 & $>0.63$ & 5 \\
\hline $160-018$ & S.. & 15.3 & Coma & 7092 & $<8.82$ & 88 & $>0.63$ & - \\
\hline $160-020$ & Pec & 15.5 & Coma & 4968 & 8.93 & 24 & 0.27 & 4 \\
\hline $160-025$ & $\mathrm{Sa}$ & 14.0 & Coma & 6702 & $<8.75$ & 27 & $>0.92$ & - \\
\hline $160-026$ & $\mathrm{Sc}$ & 15.5 & Coma & 7545 & 9.29 & 40 & 0.23 & 2 \\
\hline $160-031$ & S.. & 15.7 & Coma & 6852 & $<8.84$ & 6 & $>0.62$ & 5 \\
\hline $160-032$ & $\mathrm{Sb}$ & 14.9 & Coma & 7747 & $<8.83$ & 2 & $>0.76$ & 5 \\
\hline $160-055$ & $\mathrm{Sab}$ & 14.2 & Coma & 7164 & 9.34 & 40 & 0.49 & 2 \\
\hline $160-058$ & $\mathrm{Sbc}$ & 15.5 & Coma & 7616 & 9.49 & 126 & 0.40 & 1 \\
\hline $160-064$ & Pec & 15.4 & Coma & 7368 & $<8.32$ & 132 & $>0.93$ & - \\
\hline $160-067$ & Pec & 15.4 & Coma & 7655 & 9.34 & 181 & -0.01 & 2 \\
\hline $160-073$ & Pec & 15.1 & Coma & 5425 & 8.57 & 132 & 0.96 & 5 \\
\hline $160-076$ & $\mathrm{Sc}$ & 15.6 & Coma & 5390 & 9.64 & 132 & -0.35 & 5 \\
\hline $160-081$ & $\mathrm{Sb}$ & 14.7 & Coma & 5898 & $<8.86$ & 1 & $>1.29$ & 5 \\
\hline $160-086$ & Pec & 15.4 & Coma & 7499 & 8.74 & 126 & 0.76 & 1 \\
\hline $160-088$ & $\mathrm{Sb}$ & 14.6 & Coma & 7287 & 9.35 & 40 & 0.42 & 1 \\
\hline $160-095$ & $\mathrm{Sb}$ & 13.7 & Coma & 5482 & 9.33 & 126 & 0.96 & 1 \\
\hline $160-096 \mathrm{~N}$ & Pec & 15.2 & Coma & 6892 & 8.42 & 132 & 0.95 & 5 \\
\hline $160-098$ & Pec & 15.3 & Coma & 8762 & 9.05 & 1 & 0.41 & 2 \\
\hline $160-102$ & $\mathrm{Sab}$ & 14.8 & Coma & 7095 & 9.97 & 126 & -0.01 & 1 \\
\hline $160-106$ & Pec & 15.1 & Coma & 6876 & 9.05 & 132 & 0.54 & 5 \\
\hline $160-108$ & Pec & 15.5 & Coma & 8323 & $<8.29$ & 6 & $>0.92$ & 5 \\
\hline $160-114$ & S.. & 15.6 & Coma & 7454 & $<8.74$ & 88 & $>0.81$ & - \\
\hline $160-121$ & $\mathrm{Sb}$ & 15.5 & Coma & 6676 & 9.92 & 126 & 0.02 & 1 \\
\hline $160-127$ & $\mathrm{Sc}$ & 15.5 & Coma & 5500 & 9.71 & 126 & -0.10 & 1 \\
\hline $160-128$ & Pec & 15.3 & Coma & 7920 & 9.73 & 193 & -0.33 & 1 \\
\hline $160-137$ & $\mathrm{Sa}$ & 13.9 & Coma & 7050 & 9.79 & 40 & -0.05 & 1 \\
\hline $160-138$ & S.. & 15.7 & Coma & 7861 & $<8.56$ & 193 & $>0.71$ & - \\
\hline $160-139$ & Pec & 15.0 & Coma & 4749 & 9.96 & 126 & -0.19 & 2 \\
\hline $160-141$ & Pec & 15.5 & Coma & 7292 & 8.95 & 1 & 0.32 & 1 \\
\hline $160-146$ & $\mathrm{Sa}$ & 15.4 & Pair & 7385 & $<8.41$ & 193 & $>1.12$ & - \\
\hline $160-148$ & $\mathrm{Sa}$ & 14.3 & N5056 HL & 5988 & 9.75 & 126 & -0.07 & 1 \\
\hline $160-151$ & Pec & 15.1 & N5056 HL & 6258 & 8.97 & 24 & 0.31 & 2 \\
\hline $160-152 \mathrm{~W}$ & $\mathrm{Sb}$ & 14.0 & N5056 HL & 5610 & 9.95 & 2 & 0.05 & 1 \\
\hline $160-155$ & $\mathrm{Sb}$ & 15.3 & N5056 HL & 6366 & 9.60 & 126 & 0.04 & 1 \\
\hline $160-156$ & $\mathrm{Sa}$ & 15.3 & Pair & 7262 & 9.59 & 24 & 0.28 & 1 \\
\hline $160-163 \mathrm{~N}$ & S.. & 15.7 & Isol. & 6877 & $<8.92$ & 24 & $>0.28$ & - \\
\hline $160-164$ & $\mathrm{Sc}$ & 15.2 & N5056 HL & 6074 & 9.69 & 126 & -0.19 & 1 \\
\hline $160-166$ & $\mathrm{Sb}$ & 13.6 & N5056 HL & 6408 & 9.80 & 2 & 0.24 & 1 \\
\hline $160-167$ & $\mathrm{Sb}$ & 15.0 & N5056 HL & 6039 & 9.75 & 126 & 0.02 & 1 \\
\hline $160-168$ & $\mathrm{Sc}$ & 14.2 & Isol. & 7476 & 10.36 & 2 & -0.40 & 1 \\
\hline $160-169$ & S.. & 15.6 & Isol. & 6850 & 9.41 & 193 & 0.08 & 1 \\
\hline $160-173$ & $\mathrm{Sc}$ & 13.6 & N5056 HL & 5592 & 10.12 & 126 & -0.12 & 1 \\
\hline $160-175$ & S.. & 15.1 & N5056 HL & 5661 & 9.25 & 24 & 0.18 & 1 \\
\hline $160-180$ & S.. & 15.4 & N5056 HL & 5581 & - & - & - & - \\
\hline $160-181$ & $\mathrm{Sc}$ & 14.3 & N5056 HL & 5550 & 9.87 & 126 & -0.11 & 1 \\
\hline $160-182$ & $\mathrm{Sab}$ & 15.0 & Isol. & 6994 & 9.62 & 5 & 0.07 & 1 \\
\hline $160-183$ & Pec & 14.7 & N5056 HL & 5605 & 9.57 & 40 & -0.14 & 3 \\
\hline $160-192$ & $\mathrm{Sb}$ & 14.3 & Isol. & 6649 & 10.25 & 126 & -0.09 & 1 \\
\hline $160-195$ & S.. & 15.7 & Tripl. & 7247 & 9.14 & 193 & -0.04 & 2 \\
\hline
\end{tabular}


G. Gavazzi et al.: H I observations in the Coma Supercluster, Online Material $p 7$

Table 4. continued.

\begin{tabular}{lccccrcrr}
\hline \hline CGCG & Type & $\begin{array}{c}m_{\mathrm{p}} \\
\text { mag }\end{array}$ & Cloud & $\begin{array}{c}V \\
\mathrm{~km} \mathrm{~s}^{-1}\end{array}$ & $\begin{array}{r}\log M_{\mathrm{HI}} \\
M_{\odot}\end{array}$ & Ref. & Def $_{\mathrm{HI}}$ & Qual \\
& & & & & \\
\hline $160-206$ & $\mathrm{S..}$ & 15.6 & N5056 HL & 5053 & 8.99 & 40 & 0.25 & 1 \\
$160-207$ & $\mathrm{Sc}$ & 15.3 & N5056 HL & 5081 & 9.71 & 5 & 0.05 & 1 \\
$160-209$ & $\mathrm{Pec}$ & 15.4 & Pair & 7168 & 9.28 & 24 & 0.28 & 2 \\
$160-212$ & $\mathrm{Sa}$ & 14.9 & Coma & 7549 & 8.78 & 132 & 0.90 & 5 \\
$160-213$ & $\mathrm{Pec}$ & 15.5 & Coma & 9386 & $<8.17$ & 1 & $>1.11$ & 5 \\
$160-243$ & $\mathrm{S..}$ & 15.6 & Coma & 5121 & - & - & - & - \\
$160-252$ & $\mathrm{Pec}$ & 15.1 & Coma & 7718 & 9.01 & 40 & 0.56 & 1 \\
$160-257$ & $\mathrm{Sa}$ & 14.6 & Coma & 5821 & 8.66 & 132 & 0.97 & 5 \\
$160-260$ & $\mathrm{Sa}$ & 13.7 & Coma & 7985 & 9.18 & 126 & 0.81 & 1 \\
$160-261$ & $\mathrm{S..}$ & 15.6 & Coma & 6917 & $<8.56$ & 193 & $>0.81$ & - \\
$161-029$ & $\mathrm{Sb}$ & 15.7 & N5056 HL & 4930 & - & - & - & - \\
$161-031$ & $\mathrm{Sbc}$ & 14.9 & Tripl. & 7270 & 9.75 & 40 & 0.08 & 2 \\
$161-040$ & $\mathrm{Sc}$ & 15.6 & Isol. & 7260 & 9.43 & 40 & -0.02 & 1 \\
$161-041$ & $\mathrm{S..}$ & 15.5 & N5056 HL & 4979 & 8.81 & 88 & 0.32 & 1 \\
$161-043$ & $\mathrm{Sa}$ & 14.4 & Isol. & 6638 & 9.62 & 24 & 0.33 & 2 \\
$161-044$ & $\mathrm{Sc}$ & 15.3 & N5056 HL & 4983 & 9.21 & 40 & 0.48 & 1 \\
$161-048$ & $\mathrm{Sa}$ & 15.1 & Isol. & 7280 & $<8.82$ & 193 & $>0.72$ & - \\
$161-051$ & $\mathrm{S..}$ & 15.6 & Isol. & 7150 & $<9.17$ & 193 & $>-0.12$ & - \\
$161-052$ & $\mathrm{Pec}$ & 15.1 & Isol. & 7072 & 9.46 & 88 & -0.50 & 2 \\
$161-054$ & $\mathrm{Sa}$ & 15.5 & Isol. & 6756 & 9.41 & 193 & -0.07 & 1 \\
$161-063$ & $\mathrm{Sbc}$ & 15.5 & Isol. & 7300 & 9.97 & 88 & -0.06 & 1 \\
$161-066$ & $\mathrm{S..}$ & 15.7 & Isol. & 7380 & 10.12 & 40 & -0.56 & 3 \\
$161-069$ & $\mathrm{Sb}$ & 14.6 & Isol. & 7172 & 9.88 & 24 & -0.27 & 1 \\
$161-071$ & $\mathrm{Pec}$ & 14.9 & N5056 L & 4827 & 9.84 & 5 & -0.26 & 1 \\
$161-073$ & $\mathrm{Sb}$ & 14.2 & Isol. & 7320 & 10.38 & 24 & -0.21 & 1 \\
\hline
\end{tabular}

1: Giovanelli \& Haynes (1985); 2: Chincarini et al. (1983a); 3: Sullivan et al. (1981); 4: Chincarini et al. (1983b); 5: Fontanelli (1984); 6: Bothun et al. (1985); 7: Williams \& Kerr (1981); 15: Haynes \& Giovanelli (1984); 22: Sulentic \& Arp H (1982); 24: Gavazzi (1987); 27: Eder et al. (1991); 39: Lewis et al. (1985); 40: Gavazzi (1989); 43: Salzer et al. (1990); 88: Scodeggio \& Gavazzi (1993); 105: Lu et al. (1993); 111: Mould et al. (1995); 126: Haynes et al. (1997); 127: Dell'Antonio et al. (1996); 132: Bravo Alfaro (2001); 168: Magri et al. (1994); 181: Beijsbergen (2003); 184: van Driel et al. (2000); 185: Vogt et al. (2004); 193: This work. 


\section{Appendix A: Notes on individual galaxies}

CGCG 127-018: Our H I detection $\left(V_{\mathrm{HI}}=6922 \pm 2 \mathrm{~km} \mathrm{~s}^{-1}\right.$, $W_{50}=147 \mathrm{~km} \mathrm{~s}^{-1}$ and $I_{\mathrm{HI}}=2.1 \pm 0.06 \mathrm{Jy} \mathrm{km} \mathrm{s}^{-1}$ ) is consistent with our previous (van Driel et al. 2000) Nançay detection $\left(V_{\mathrm{HI}}=6936 \pm 16 \mathrm{~km} \mathrm{~s}^{-1}, W_{50}=131 \mathrm{~km} \mathrm{~s}^{-1}\right.$ and $\left.I_{\mathrm{HI}}=2.7 \pm 0.3 \mathrm{Jy} \mathrm{km} \mathrm{s}^{-1}\right)$ and with the Arecibo detection of Gavazzi (1987) at $V_{\mathrm{HI}}=6935 \mathrm{~km} \mathrm{~s}^{-1}$.

CGCG 127-039: The integrated line intensity of our $\mathrm{HI}$ detection $\left(V_{\mathrm{HI}}=6911 \pm 1 \quad \mathrm{~km} \mathrm{~s}^{-1}, \quad W_{50}=37 \quad \mathrm{~km} \mathrm{~s}^{-1}\right.$ and $I_{\mathrm{HI}}=0.82 \pm 0.04 \mathrm{Jy} \mathrm{km} \mathrm{s}^{-1}$ ) is 3.4 times lower than that of our previous Nançay detection (van Driel et al. 2000), with $V_{\mathrm{HI}}=6919 \pm 9 \mathrm{~km} \mathrm{~s}^{-1}, W_{50}=40 \mathrm{~km} \mathrm{~s}^{-1}$ and $I_{\mathrm{HI}}=2.8 \pm 0.2 \mathrm{Jy} \mathrm{km} \mathrm{s}^{-1}$. The latter was made with an elongated $3.6 \times 21^{\prime}(\alpha \times \delta) H P B W$, which is considerably larger than the 3'6 round Arecibo beam. This difference can be due to the 13.6 B mag SBbc spiral NGC 3832, 17 '.2 due south of the target galaxy, whose mean HI line parameters $\left(V_{\mathrm{HI}}=6909 \pm 6 \mathrm{~km} \mathrm{~s}^{-1}, W_{50}=171 \mathrm{~km} \mathrm{~s}^{-1}\right.$ and $\left.I_{\mathrm{HI}}=10.4 \mathrm{Jy} \mathrm{km} \mathrm{s}^{-1}\right)$ are based on 5 spectra, all obtained at Arecibo (Chincarini et al. 1983a; Giovanardi \& Salpeter 1985; Lewis 1985; Lewis et al. 1985; Sullivan et al. 1981).

CGCG 127-055: Our H I detection $\left(V_{\mathrm{HI}}=6626 \pm 3 \mathrm{~km} \mathrm{~s}^{-1}\right.$, $W_{50}=215 \mathrm{~km} \mathrm{~s}^{-1}$ and $\left.I_{\mathrm{HI}}=1.9 \pm 0.08 \mathrm{Jy} \mathrm{km} \mathrm{s}^{-1}\right)$ is consistent with our previous measurement (van Driel et al. 2000) made at Nançay, with $V_{\mathrm{HI}}=6656 \pm 21 \mathrm{~km} \mathrm{~s}^{-1}, W_{50}=183 \mathrm{~km} \mathrm{~s}^{-1}$ and $I_{\mathrm{HI}}=2.1 \pm 0.3 \mathrm{Jy} \mathrm{km} \mathrm{s}^{-1}$. Our new data have a 3.6 times better rms noise level.

CGCG 128-072: Given the uncertainties, the global parameters of our $\mathrm{HI}$ detection $\left(V_{\mathrm{HI}}=6795 \pm 6 \mathrm{~km} \mathrm{~s}^{-1}\right.$, $W_{50}=119 \mathrm{~km} \mathrm{~s}^{-1}$ and $\left.I_{\mathrm{HI}}=0.82 \pm 0.07 \mathrm{Jy} \mathrm{km} \mathrm{s}^{-1}\right)$ are consistent with those of our previous Nançay detection (van Driel et al. 2000), with $V_{\mathrm{HI}}=6848 \pm 81 \mathrm{~km} \mathrm{~s}^{-1}, W_{50}=213 \mathrm{~km} \mathrm{~s}^{-1}$ and $I_{\mathrm{HI}}=1.4 \pm 0.6 \mathrm{Jy} \mathrm{km} \mathrm{s}^{-1}$. Our new data have a 5.4 times better rms noise level.

CGCG 129-004: discrepant values for the optical redshift have been published, 4847 and $6729 \mathrm{~km} \mathrm{~s}^{-1}$ (Gavazzi et al. 1999a, and the compilation by Falco et al. 1999); we find an $\mathrm{HI}$ value of $6736 \pm 7 \mathrm{~km} \mathrm{~s}^{-1}$, consistent with the latter value.
CGCG 130-003: there are two discrepant literature values for its optical redshift, 7094 and 22,425 $\mathrm{km} \mathrm{s}^{-1}$ (Straus et al. 1992; Gregory et al. 1988); we find an Hi value of $7140 \pm 4 \mathrm{~km} \mathrm{~s}^{-1}$, consistent with the former value.

CGCG 157-044: our H I detection $\left(V_{\mathrm{HI}}=6607 \pm 4 \mathrm{~km} \mathrm{~s}^{-1}\right.$, $W_{50}=240 \mathrm{~km} \mathrm{~s}^{-1}$ and $I_{\mathrm{HI}}=0.84 \pm 0.08 \mathrm{Jy} \mathrm{km} \mathrm{s}^{-1}$ ) has a 1.7 times lower line intensity than our previous Nançay detection (van Driel et al. 2000), with $V_{\mathrm{HI}}=6628 \pm 36 \mathrm{~km} \mathrm{~s}^{-1}$, $W_{50}=309 \mathrm{~km} \mathrm{~s}^{-1}$ and $I_{\mathrm{HI}}=1.5 \pm 0.4 \mathrm{Jy} \mathrm{km} \mathrm{s}^{-1}$. The difference is less than two times the uncertainty in the latter value, however, and therefore not significant.

CGCG 159-071: the global parameters of our Hi detection $\left(V_{\mathrm{HI}}=6971 \pm 1 \mathrm{~km} \mathrm{~s}^{-1}\right.$ and $W_{50}=189 \mathrm{~km} \mathrm{~s}^{-1}$ and $I_{\mathrm{HI}}=2.6 \pm 0.1 \mathrm{Jy} \mathrm{km} \mathrm{s}{ }^{-1}$ ) are consistent with those of our previous Nançay detection (van Driel et al. 2000), with $V_{\mathrm{HI}}=6985 \pm 6 \mathrm{~km} \mathrm{~s}^{-1}, W_{50}=164 \mathrm{~km} \mathrm{~s}^{-1}$ and $I_{\mathrm{HI}}=2.4 \pm 0.4 \mathrm{Jy} \mathrm{km} \mathrm{s}^{-1}$.

CGCG 159-097: Its optical redshift, $6573 \pm 190 \mathrm{~km} \mathrm{~s}^{-1}$, is not well determined. We measured an $\mathrm{HI}$ value of $6424 \pm 30 \mathrm{~km} \mathrm{~s}^{-1}$, consistent with 3 of the published optical values - only the optical velocity of $6883 \pm 75 \mathrm{~km} \mathrm{~s}^{-1}$ measured by van Haarlem et al. (1993) is in disagreement with all other values.

CGCG 160-128: Our detection $\left(V_{\mathrm{HI}}=7920 \pm 1 \mathrm{~km} \mathrm{~s}^{-1}\right.$, $W_{50}=115 \mathrm{~km} \mathrm{~s}^{-1}$ and $\left.I_{\mathrm{HI}}=2.5 \pm 0.07 \mathrm{Jy} \mathrm{km} \mathrm{s}^{-1}\right)$ is consistent with our previous Nançay detection $\left(V_{\mathrm{HI}}=7940 \pm 5 \mathrm{~km} \mathrm{~s}^{-1}\right.$, $W_{50}=100 \mathrm{~km} \mathrm{~s}^{-1}$ and $I_{\mathrm{HI}}=2.2 \pm 0.3 \mathrm{Jy} \mathrm{km} \mathrm{s}^{-1}$ ), which was based on data with a 4 times higher rms of $2.8 \mathrm{mJy}$ (van Driel et al. 2000).

CGCG 161-051: We did not confirm our previous, quite tentative Nançay detection (van Driel et al. 2000), with $V_{\mathrm{HI}}=6993: \mathrm{km} \mathrm{s}^{-1}, W_{50}=235: \mathrm{km} \mathrm{s}^{-1}$ and $I_{\mathrm{HI}}=1.5: \mathrm{Jy} \mathrm{km} \mathrm{s}^{-1}$. Our new spectrum has a 2.4 times better rms, of $1.5 \mathrm{mJy}$.

CGCG 161-054: Our detection $\left(V_{\mathrm{HI}}=6756 \pm 3 \mathrm{~km} \mathrm{~s}^{-1}\right.$, $W_{50}=284 \mathrm{~km} \mathrm{~s}^{-1}$ and $\left.I_{\mathrm{HI}}=1.3 \pm 0.1 \mathrm{Jy} \mathrm{km} \mathrm{s}^{-1}\right)$ is consistent with our previous, tentative Nançay detection, with $V_{\mathrm{HI}}=6760: \mathrm{km} \mathrm{s}^{-1}, W_{50}=335: \mathrm{km} \mathrm{s}^{-1}$ and $I_{\mathrm{HI}}=1.8: \mathrm{Jy} \mathrm{km} \mathrm{s}^{-1}$, which was based on data with a 4 times higher rms of $3.2 \mathrm{mJy}$ (van Driel et al. 2000). 
G. Gavazzi et al.: H I observations in the Coma Supercluster, Online Material p 9
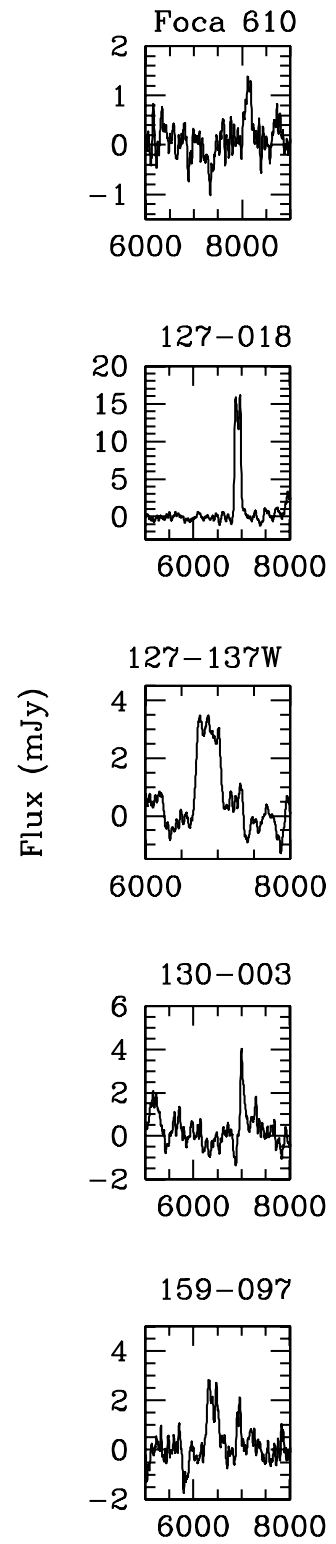
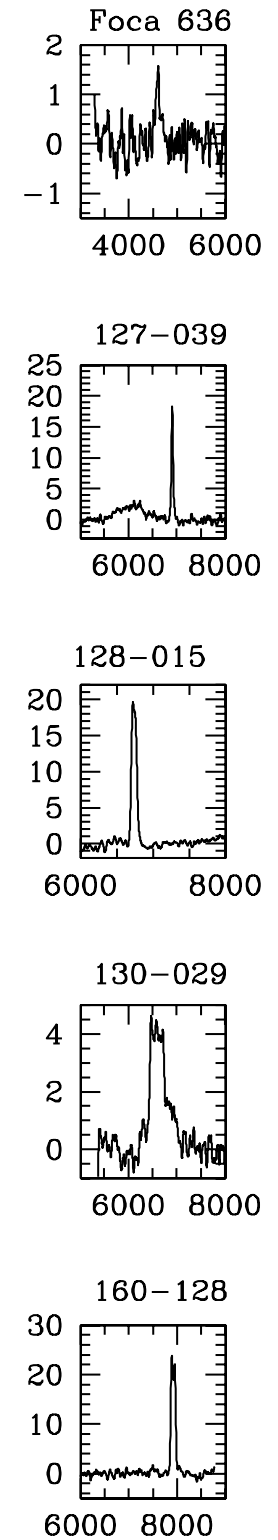
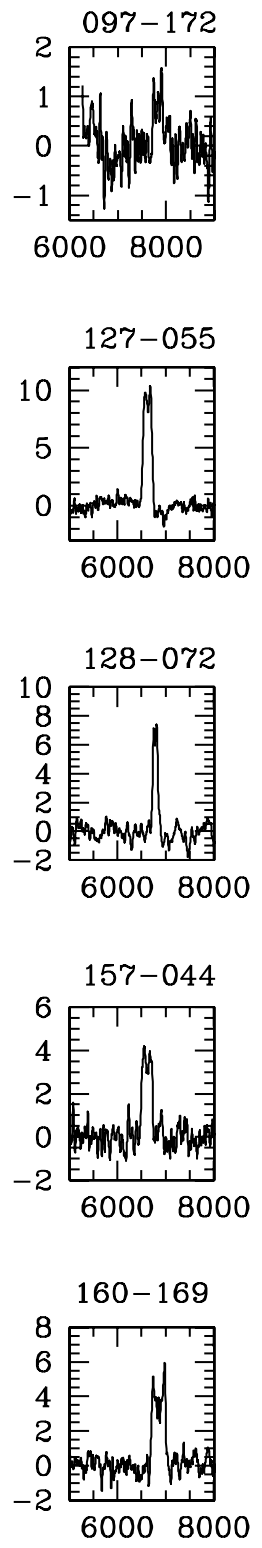

$$
\text { Vel }\left(\mathrm{km} \mathrm{s}^{-1}\right)
$$
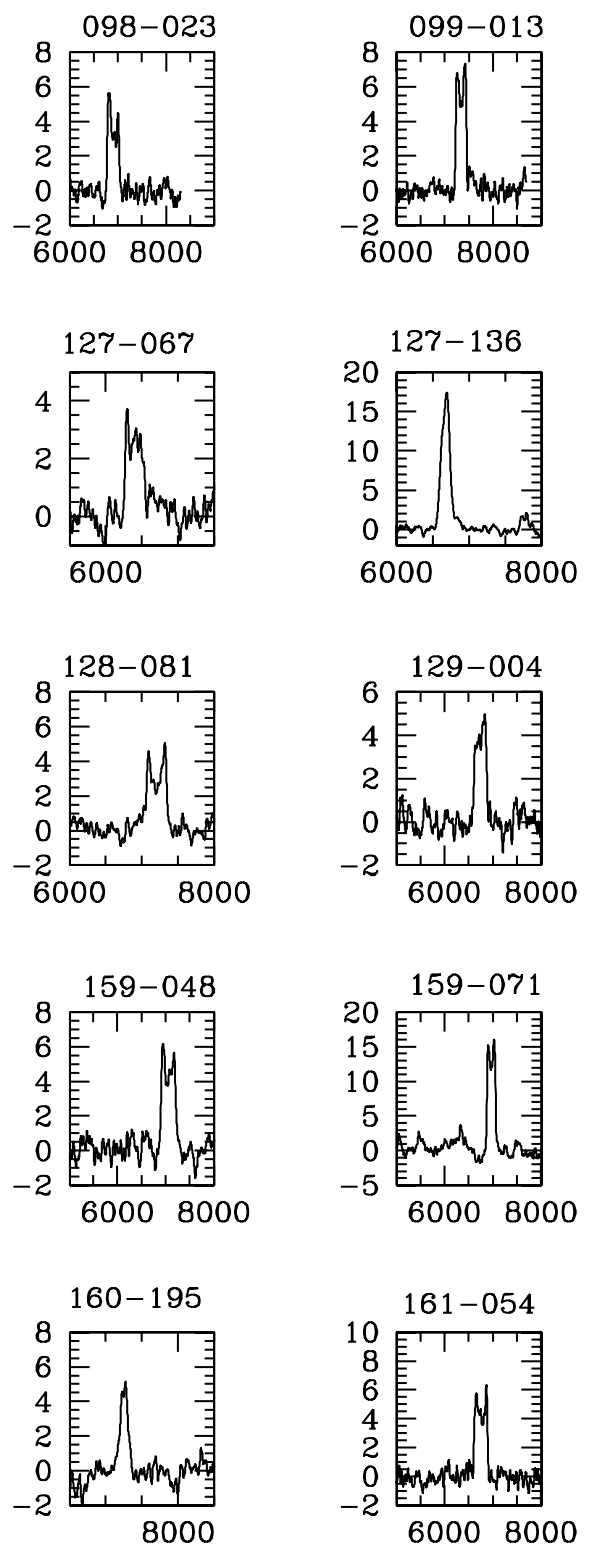

Fig. A.1. H I spectra of the tentatively detected galaxies in the Coma Supercluster. 
G. Gavazzi et al.: H I observations in the Coma Supercluster, Online Material p 10
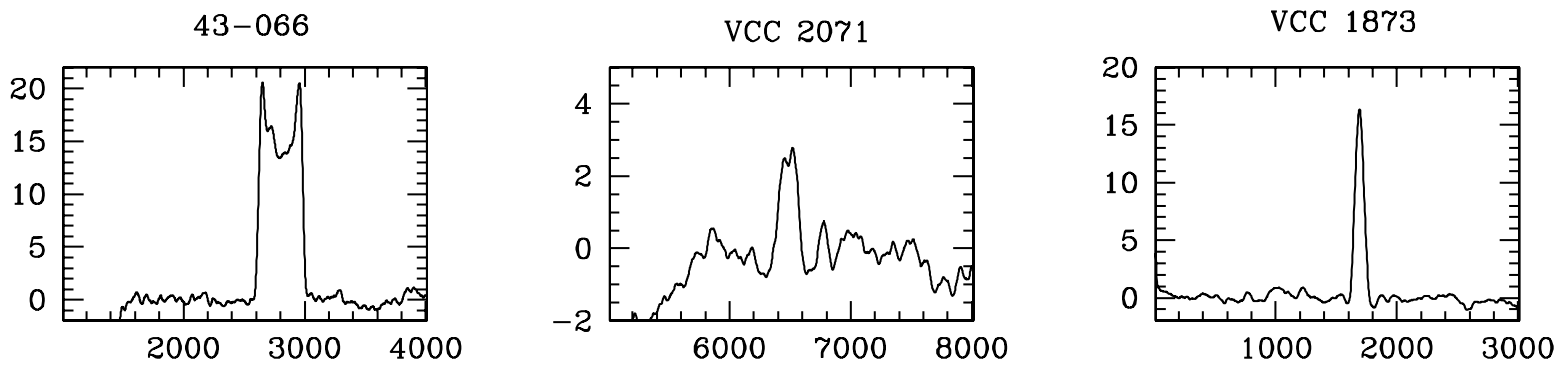

VCC 1821
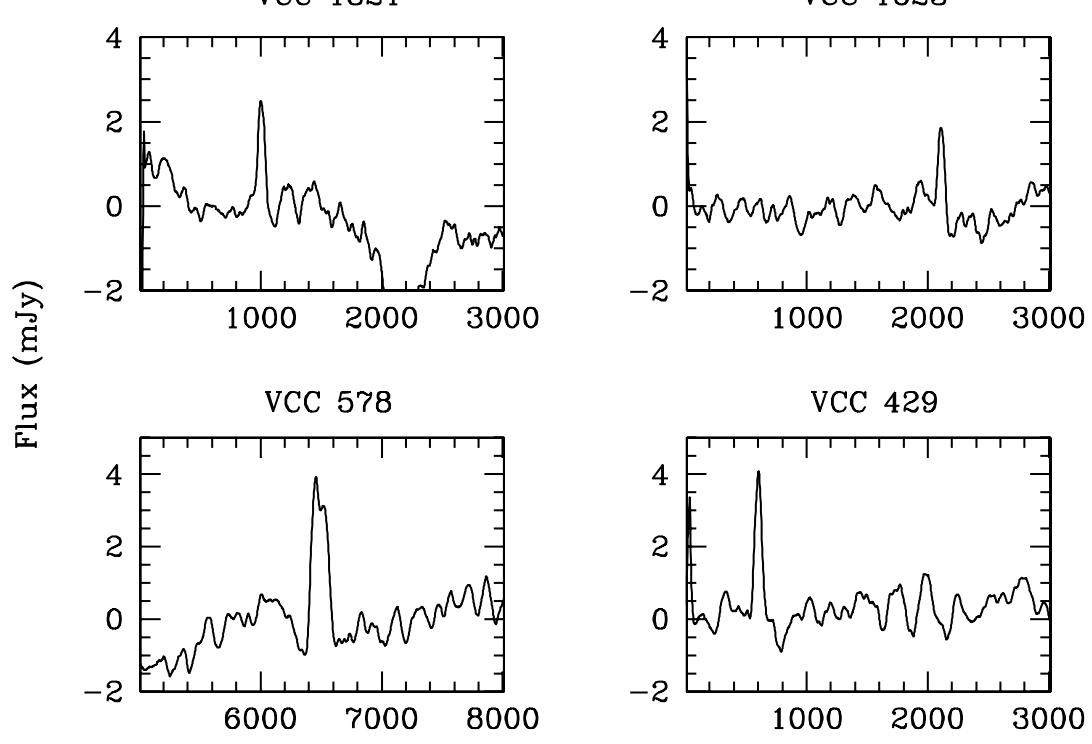

VCC 1574

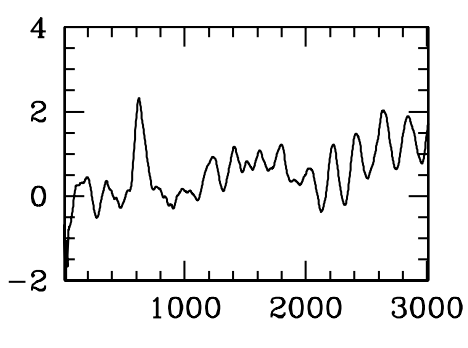

VCC 429

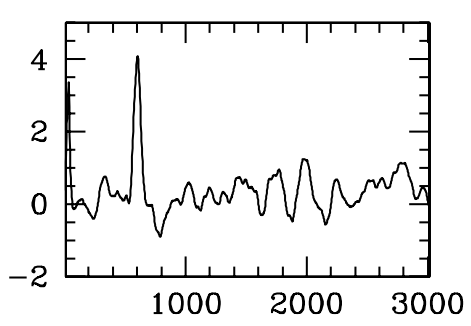

VCC 137

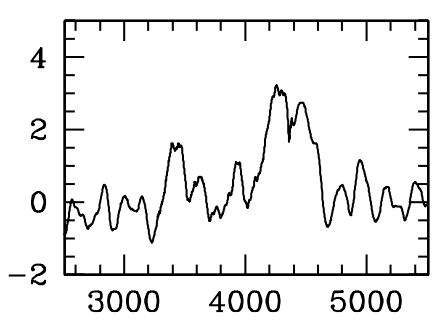

VCC 85
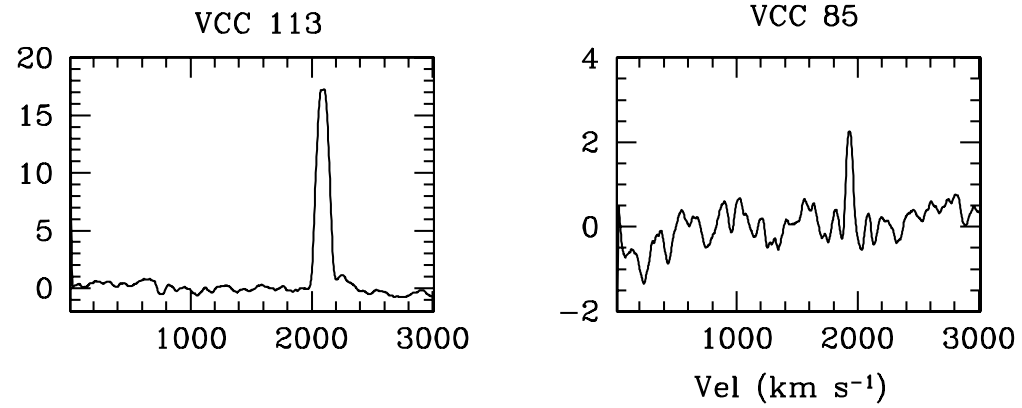

VCC 30

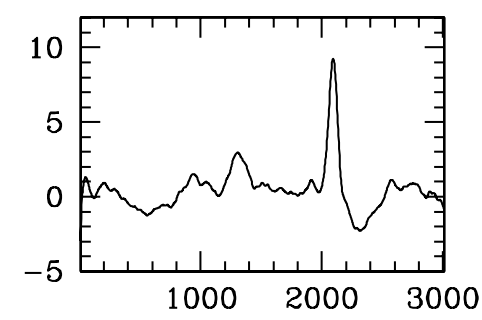

Fig. A.2. H I spectra of the tentatively detected galaxies in the Virgo cluster. 